\title{
Algebras of p-adic distributions and admissible representations
}

\author{
P. Schneider, J. Teitelbaum
}

\section{Introduction}

In a series of earlier papers, ([ST1-4]) we began a systematic study of locally analytic representations of a locally $L$-analytic group $G$, where $L \subseteq \mathbb{C}_{p}$ is a finite extension of $\mathbb{Q}_{p}$. Such a representation is given by a continuous action of $G$ on a locally convex topological vector space $V$ over a spherically complete extension field $K \subseteq \mathbb{C}_{p}$ of $L$, such that the orbit maps $g \mapsto g v$ are locally analytic functions on $G$. When $G$ is the group of $L$-points of an algebraic group, the class of such representations includes many interesting examples, such as the principal series representations studied in [ST2], the finite dimensional algebraic representations, and the smooth representations of Langlands theory. A reasonable theory of such representations requires the identification of a finiteness condition that is broad enough to include the important examples and yet restrictive enough to rule out pathologies. In this paper we present such a finiteness condition that we call "admissibility" for locally analytic representations (provided the field $K$ is discretely valued). The admissible locally analytic representations, among which are the examples mentioned above, form an abelian category.

Our approach to the characterization of admissible representations is based on the algebraic approach to such representations begun in [ST2]. As in that paper, we require that the vector space $V$ carrying the locally analytic representation be of compact type, a topological condition whose most important consequence is that $V$ is reflexive. We focus our attention on the algebra $D(G, K)$ of locally analytic distributions on $G$. This algebra is the continuous dual of the locally analytic, $K$-valued functions on $G$, with multiplication given by convolution. When $G$ is compact, $D(G, K)$ is a Fréchet algebra, but in general is neither noetherian nor commutative. If $V$ is a locally analytic $G$-representation, then its continuous dual $V_{b}^{\prime}$, with its strong topology, becomes a module over $D(G, K)$. We identify a subcategory of the module category of $D(G, K)$ that we call the coadmissible modules. We show that any coadmissible module carries a canonical Fréchet topology. We say that $V$ is admissible if $V_{b}^{\prime}$ is topologically isomorphic to a coadmissible module. If $G$ is not compact, we say that $V$ is admissible if it is admissible as a representation for one (or equivalently any) compact open subgroup of $G$.

To define the category of coadmissible $D(G, K)$-modules, we introduce a general class of Fréchet algebras that we call Fréchet-Stein algebras. The definition of a Fréchet-Stein algebra, and its associated category of coadmissible modules, is inspired by the notion of a Stein algebra, the (commutative) ring of functions on a Stein space, as described in [For]. We recall that for the commutative 
group $G=\mathbb{Z}_{p}^{d}$ the algebra $D\left(G, \mathbb{Q}_{p}\right)$ is naturally isomorphic, via Fourier theory, to the ring of rigid analytic functions $\mathcal{O}(\mathcal{X})$ on the locally analytic character group $\mathcal{X}=\mathcal{X}\left(\mathbb{Z}_{p}^{d}\right)$ of $\mathbb{Z}_{p}^{d}$. This latter space $\mathcal{X}$ is the $d$-dimensional open unit disk over $\mathbb{Q}_{p}$. On a Stein space like $\mathcal{X}$ the category of coherent module sheaves is a well behaved abelian category which, under the global section functor, is equivalent to an abelian subcategory of the category $\operatorname{Mod}(\mathcal{O}(\mathcal{X}))$ of all $\mathcal{O}(\mathcal{X})$ modules. A closer inspection of the proofs of these facts reveals that, truly in the spirit of noncommutative geometry, they are consequences of certain basic properties of the ring $\mathcal{O}(\mathcal{X})$ which can be formulated without making use of the actual geometric object $\mathcal{X}$ and which do not even require the commutativity of the ring. From this observation we are led to define a Fréchet-Stein algebra to be, roughly speaking, a Fréchet algebra that is a projective limit of noetherian Banach algebras, where the transition maps in the projective system are flat. A module over a Fréchet-Stein algebra is coadmissible if it is the projective limit of a compatible system of finitely generated modules over the associated Banach algebras. The category of coadmissible modules for a Fréchet-Stein algebra is abelian, and includes for example all finitely presented $D(G, K)$-modules.

With the general notion of Fréchet-Stein algebra in hand, our first main result is that, when $G$ is compact, the algebra $D(G, K)$ is Fréchet-Stein. Our proof relies heavily on Lazard's detailed study of $p$-adic analytic groups in his classic work [Laz], and on the graded ring techniques presented in the book [LVO]. The idea is that a sufficiently small $p$-valued group is isomorphic as a $p$-adic manifold to $\mathbb{Z}_{p}^{d}$, and so the vector space underlying the algebra $D(G, K)$ is isomorphic to the dual of the $K$-valued locally analytic functions on $\mathbb{Z}_{p}^{d}$. This space is known explicitly through the theory of Mahler expansions. However, the multiplication on $D(G, K)$ comes from the non-commutative multiplication on $G$. We use graded techniques and Lazard's results to pass from commutative to noncommutative information.

The idea to construct these Banach algebras appears for the first time in the thesis of H. Frommer on the locally analytic principal series of $G L_{n}$. He deals, by methods quite different from ours, with the special case of the group $G$ of all unipotent triangular matrices in $G L_{n}\left(\mathbb{Z}_{p}\right)$.

The Fréchet-Stein property of $D(G, K)$ makes it possible to define admissible representations as outlined above. Another consequence is that any finitely generated submodule of a finitely generated free $D(G, K)$-module is closed. It follows that any finitely presented $D(G, K)$-module is analytic in the sense of [ST1], answering a question raised in that paper.

Our second main result concerns the relation between the completed group ring $\mathbb{Z}_{p}[[G]]$ of a compact $G$, studied in detail by Lazard in [Laz], and the algebra $D(G, K)$. In topological terms, the ring $L[[G]]:=L \otimes \mathbb{Z}_{p}[[G]]$ is the Iwasawa algebra of "measures", and is dual to the $L$-valued continuous functions on $G$. Because the locally analytic functions are a subspace of the continuous functions, 
there is a natural map $L[[G]] \rightarrow D(G, K)$. When $G$ is compact and locally $\mathbb{Q}_{p^{-}}$ analytic, we prove that this map is faithfully flat, answering a question raised in [ST3]. This result implies that, in an admissible continuous representation of such a $G$ on a Banach space, as studied in [ST3], the subspace of locally analytic vectors is dense, and "passage to analytic vectors" is an exact functor.

The final topic of this paper is the development of a dimension theory for coadmissible modules over $D(G, K)$. We begin by considering such a theory for a general Fréchet-Stein algebra $A$. Our approach is modelled on the dimension theory of coherent sheaves on Stein spaces as presented in [Ban]. In order to have a well-defined "local" notion of dimension, we require that the Banach algebras defining the Fréchet-Stein structure of $A$ be Auslander regular rings of bounded global dimension. Under this assumption one can adopt the point of view that the "local codimension of the support of a sheaf" is measured by the grade of the module over the Banach algebra. We then show that correspondingly the grade of a coadmissible module over $A$ has the usual properties of a "global" codimension.

Having developed these general properties of codimension, we use graded techniques and our earlier results to show that $D(G, K)$ satisfies the necessary regularity conditions when $G$ is a compact, $d$-dimensional, $\mathbb{Q}_{p}$-analytic group. Consequently every coadmissible module over $D(G, K)$ has a well-defined codimension bounded above by $d$, as well as a dimension filtration by coadmissible submodules. We also will show that, if $V$ is a smooth admissible representation, or more generally a $U(\mathfrak{g})$-finite admissible representation as in [ST1], then the dual $D(G, K)$-module $V_{b}^{\prime}$ has maximal codimension equal to $d$.

We begin the paper with a brief review of filtered and graded techniques. After some preliminaries on Banach algebras, we define Fréchet-Stein algebras and their coadmissible modules and establish the important properties of the category of such modules in section 3. Then we turn to $D(G, K)$, and after reviewing some of Lazard's key results from [Laz] we prove the two main results on $D(G, K)$ in sections 4 and 5 . The application to representation theory follows in section 6 . Among other results we show that all smooth admissible representations in the sense of Langlands theory are admissible locally analytic representations. In fact the former can be characterized among the latter by a trivial derived action of the Lie algebra of $G$. In the final two sections we discuss the existence of analytic vectors in continuous representations, and develop the dimension theory of coadmissible modules.

Acknowledgements: The second author was supported by a grant from the US National Security Agency Mathematical Sciences Program. Both authors gratefully acknowledge support from the University of Illinois at Chicago and from the SFB "Geometrische Strukturen in der Mathematik" at Münster making possible a series of mutual visits. 
Notation: For any associative unital ring $R$ we let $\mathcal{M}_{R}$ denote the full subcategory of all finitely generated $R$-modules in the category $\operatorname{Mod}(R)$ of all (left) unital $R$-modules. If $R$ is (left) noetherian then $\mathcal{M}_{R}$ is an abelian category.

Throughout $K$ is a field which is complete with respect to a non-trivial nonarchimedean absolute value || . For the basic notions and facts in nonarchimedean functional analysis we refer to [NFA].

Finally, $p$ is a fixed prime number.

\section{Filtered rings}

If a ring is filtered then, quite often, it inherits properties of the associated graded ring. Since this technique will be of fundamental importance for us later on we begin by recalling some of it.

Throughout this section let $R$ be an associative unital ring. We call $R$ filtered if it is equipped with a family $\left(F^{s} R\right)_{s \in \mathbb{R}}$ of additive subgroups $F^{s} R \subseteq R$ such that, for any $r, s \in \mathbb{R}$,

$-F^{r} R \supseteq F^{s} R$ if $r \leq s$,

$-F^{r} R \cdot F^{s} R \subseteq F^{r+s} R$,

- $\bigcup_{s \in \mathbb{R}} F^{s} R=R$ and $1 \in F^{0} R$.

For any $s \in \mathbb{R}$ put

$$
F^{s+} R:=\bigcup_{r>s} F^{r} R \quad \text { and } \quad g r^{s} R:=F^{s} R / F^{s+} R .
$$

Then

$$
g r \cdot R:=\bigoplus_{s \in \mathbb{R}} g r^{s} R
$$

with the obvious multiplication is called the associated graded ring. The filtration is called quasi-integral if there exists an $n_{0} \in \mathbb{N}$ such that $\left\{s \in \mathbb{R}: g r^{s} R \neq\right.$ $0\} \subseteq \mathbb{Z} \cdot 1 / n_{0}$. Usually we also assume that the filtered ring $R$ is (separated and) complete, i.e., that the natural map

$$
R \stackrel{\cong}{\longleftarrow} \lim _{s} R / F^{s} R
$$

is bijective.

Proposition 1.1: Let $R$ be a complete filtered ring whose filtration is quasiintegral; if the graded ring gr $R$ is (left) noetherian then $R$ is (left) noetherian as well. 
Proof: Up to rescaling and reversing to increasing filtrations this is [LVO] Prop. I.7.1.2.

Any homomorphism $\phi: R \longrightarrow A$ between filtered rings which respects the filtrations induces in the obvious way a homomorphism of graded rings $g r^{*} \phi$ : $g r \cdot R \longrightarrow g r \cdot A$.

In the following we consider two complete filtered rings $R$ and $A$ whose filtrations are quasi-integral and a unital ring homomorphism $\phi: R \longrightarrow A$ which respects the filtrations.

Proposition 1.2: Suppose that $g r^{\cdot} R$ and $g r^{\cdot} A$ are left noetherian and that $g r^{*} A$ as a right $\mathrm{gr}^{\cdot} R$-module (via $\mathrm{gr}^{\cdot} \phi$ ) is flat; then $A$ is flat as a right $R$-module (via $\phi)$.

Proof: This is a combination of results in [LVO]. By Prop. II.1.2.1 it suffices to check that

(a) good filtrations on filtered $A$-modules are separated and

(b) $F \cdot R$ has the left Artin-Rees property.

According to Thm. I.5.7 the filtration $F^{\cdot} R$ induces a good filtration on any left ideal in $R$. Hence, by Remark II.1.1.2(1), we have property (b). Similarly, of course, $F^{\cdot} A$ has the Artin-Rees property. Then, by Thm. II.1.1.5, the Rees ring $\widetilde{A}$ is left noetherian which, by Lemma I.3.5.5(2) and Cor. I.5.5(3), implies property (a).

We will need a few intermediate steps in the above proofs as results in their own right.

Let $J \subseteq R$ be a left ideal equipped with the filtration induced by $F^{\cdot} R$. Similarly we equip the ideal $A J$ in $A$ with the filtration induced by $F^{\cdot} A$. Considering on $A \otimes_{R} J$ the tensor product filtration we then have the natural graded maps

$$
g r^{\cdot} A \otimes_{g r^{\cdot} R} g r^{\cdot} J \longrightarrow g r^{\cdot}\left(A \otimes_{R} J\right) \longrightarrow g r^{\cdot} A J
$$

of which the left one is surjective by construction.

Lemma 1.3: Suppose that $g r^{\cdot} R$ and $g r^{\cdot} A$ are left noetherian and that $g r^{\cdot} A$ as a right $\mathrm{gr}^{\prime} R$-module is flat; then

$$
g r^{\cdot} A \otimes_{g r \cdot R} g r \cdot \stackrel{\cong}{\longrightarrow} g r^{\cdot}\left(A \otimes_{R} J\right) \stackrel{\cong}{\longrightarrow} g r^{\cdot} A J .
$$

Proof: By the flatness assumption the first and the third term in the assertion both are submodules of $g r^{*} A \otimes_{g r^{\cdot} R} g r^{\cdot} R=g r^{*} A$. It follows that $g r^{*} A \otimes_{g r^{\cdot} R}$ 
$g r^{\prime} J \cong g r^{\cdot}\left(A \otimes_{R} J\right)$ and that $g r^{\cdot}\left(A \otimes_{R} J\right) \hookrightarrow g r^{\cdot} A J$ is injective. Using [LVO] Cor. I.4.2.5 we may conclude that the latter map even is an isomorphism provided the tensor product filtration on $A \otimes_{R} J$ is separated. But this follows from [LVO] Lemma I.6.6.15 and Prop. II.1.2.3 which, in particular, say that the tensor product $A \otimes_{R}$. preserves good filtrations and that good filtrations (for $A$ ) are separated.

Lemma 1.4: Suppose that $\mathrm{gr}^{\cdot} R$ is left noetherian; there are nonzero elements $a_{1}, \ldots, a_{l} \in J$, say $a_{i} \in F^{s_{i}} J \backslash F^{s_{i}+} J$, such that

$$
F^{s} J=\sum_{i=1}^{l} F^{s-s_{i}} R \cdot a_{i}
$$

for any $s \in \mathbb{R}$.

Proof: This is contained in the proof of [LVO] Thm. I.5.7.

\section{Preliminaries on Banach algebras}

A $K$-Banach algebra $A$ is a $K$-Banach space $\left(A,||_{A}\right)$ with a structure of an associative unital $K$-algebra such that the multiplication is continuous, which means that there is a constant $c>0$ such that

$$
|a b|_{A} \leq c|a|_{A}|b|_{A} \quad \text { for any } a, b \in A
$$

The norm will be called submultiplicative if it satisfies the stronger condition that

$$
|1|_{A}=1 \quad \text { and } \quad|a b|_{A} \leq|a|_{A}|b|_{A} \quad \text { for any } a, b \in A
$$

Proposition 2.1: Suppose that $A$ is a (left) noetherian K-Banach algebra; we then have:

i. Each module $M$ in $\mathcal{M}_{A}$ carries a unique $K$-Banach space topology (called its canonical topology) such that the A-module structure map $A \times M \rightarrow M$ is continuous;

ii. every A-submodule of a module in $\mathcal{M}_{A}$ is closed in the canonical topology; in particular, every (left) ideal in $A$ is closed;

iii. any map in $\mathcal{M}_{A}$ is continuous and strict for the canonical topologies.

Proof: The arguments in [BGR] 3.7.2 and 3.7.3 generalize in a straightforward way to the noncommutative setting. We point out that, by [BGR] Prop. 1.2.1/2, the given norm always can be replaced by an equivalent one which is submultiplicative. 
In case the norm ||$_{A}$ is submultiplicative we may and will view the $K$-Banach algebra $A$ as a complete filtered ring with respect to the filtration

$$
F^{s} A:=\left\{a \in A:|a|_{A} \leq p^{-s}\right\} .
$$

In general this filtration, of course, is not quasi-integral.

\section{Fréchet-Stein algebras}

The following is very much inspired by the notions of a Stein algebra and a Stein module in complex analysis as presented in [For].

Let $A$ be a $K$-Fréchet algebra which means that $A$ is an associative unital $K$-algebra such that the underlying $K$-vector space has the structure of a $K$ Fréchet space and such that the algebra multiplication is continuous. Consider a continuous seminorm on $A$. It induces a norm on the quotient space $A /\{a \in$ $A: q(a)=0\}$. The completion of the latter with respect to $q$ is a $K$-Banach space which will be denoted by $A_{q}$. It comes with a natural continuous linear map $A \rightarrow A_{q}$ with dense image. For any two continuous seminorms $q^{\prime} \leq q$ the identity on $A$ extends to a continuous, in fact norm decreasing, linear map $\phi_{q}^{q^{\prime}}: A_{q} \longrightarrow A_{q^{\prime}}$ with dense image such that the diagram

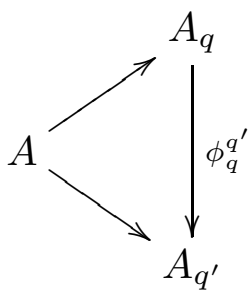

commutes. For any sequence $q_{1} \leq q_{2} \leq \ldots \leq q_{n} \leq \ldots$ of seminorms on $A$ which define the Fréchet topology (such a sequence always exists), the obvious map

$$
A \stackrel{\cong}{\underset{n \in \mathbb{N}}{\lim }} A_{q_{n}}
$$

where on the right hand side the projective limit is formed with respect to the $\phi_{q_{n+1}}^{q_{n}}$ as the transition maps is an isomorphism of locally convex $K$-vector spaces. In the following a continuous seminorm $q$ on $A$ will be called an algebra seminorm if the multiplication on $A$ is continuous with respect to $q$, i.e., if there is a constant $c>0$ such that

$$
q(a b) \leq c q(a) q(b) \text { for any } a, b \in A
$$

In this case $A_{q}$ in a natural way is a $K$-Banach algebra and the natural map $A \rightarrow A_{q}$ is a homomorphism of $K$-algebras. If the sequence $q_{1} \leq \ldots \leq q_{n} \leq$ 
... consists of algebra seminorms then the transition maps $\phi_{q_{n+1}}^{q_{n}}$ are algebra homomorphisms and

$$
A \stackrel{\cong}{\underset{n \in \mathbb{N}}{\lim }} A_{q_{n}}
$$

is an isomorphism of Fréchet algebras.

Definition: The $K$-Fréchet algebra $A$ is called a $K$-Fréchet-Stein algebra if there is a sequence $q_{1} \leq \ldots \leq q_{n} \leq \ldots$ of continuous algebra seminorms on $A$ which define the Fréchet topology such that

(i) $A_{q_{n}}$ is (left) noetherian, and

(ii) $A_{q_{n}}$ is flat as a right $A_{q_{n+1}}$-module (via $\phi_{q_{n+1}}^{q_{n}}$ )

for any $n \in \mathbb{N}$.

Fix in the following a $K$-Fréchet-Stein algebra $A$ and a sequence $\left(q_{n}\right)_{n \in \mathbb{N}}$ as in the above definition. We want to introduce the notions of coherent sheaves and coadmissible modules for $A$.

Definition: $A$ coherent sheaf for $\left(A,\left(q_{n}\right)\right)$ is a family $\left(M_{n}\right)_{n \in \mathbb{N}}$ of modules $M_{n}$ in $\mathcal{M}_{A_{q_{n}}}$ together with isomorphisms $A_{q_{n}} \otimes_{A_{q_{n+1}}} M_{n+1} \stackrel{\cong}{\longrightarrow} M_{n}$ in $\mathcal{M}_{A_{q_{n}}}$ for any $n \in \mathbb{N}$.

The coherent sheaves for $\left(A,\left(q_{n}\right)\right)$ with the obvious notion of a homomorphism

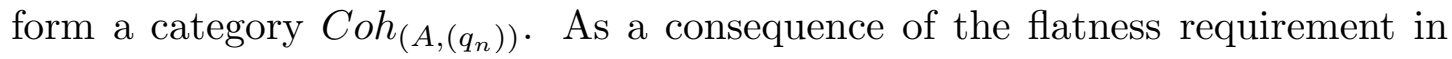
the definition of Fréchet-Stein this category is abelian, again with the obvious notions of (co)kernels and (co)images. For any coherent sheaf $\left(M_{n}\right)_{n}$ for $\left(A,\left(q_{n}\right)\right)$ its $A$-module of "global sections" is defined by

$$
\Gamma\left(M_{n}\right):=\lim _{n} M_{n}
$$

Definition: A (left) A-module is called coadmissible if it is isomorphic to the module of global sections of some coherent sheaf for $\left(A,\left(q_{n}\right)\right)$.

We let $\mathcal{C}_{A}$ denote the full subcategory of coadmissible modules in the category $\operatorname{Mod}(A)$. A simple cofinality argument shows that $\mathcal{C}_{A}$ indeed is independent of the choice of the sequence $\left(q_{n}\right)_{n}$. Passing to global sections defines a functor

$$
\Gamma: \operatorname{Coh}_{\left(A,\left(q_{n}\right)\right)} \longrightarrow \mathcal{C}_{A}
$$


Theorem: Let $\left(M_{n}\right)_{n}$ be a coherent sheaf for $\left(A,\left(q_{n}\right)\right)$ and put $M:=\Gamma\left(M_{n}\right)$; we have:

i. (Theorem A) For any $n \in \mathbb{N}$ the natural map $M \longrightarrow M_{n}$ has dense image with respect to the canonical topology on the target;

ii. (Theorem B)

$$
{\underset{\lim }{n}}^{(i)} M_{n}=0
$$

for any natural number $i \geq 1$.

Proof: i. Fix an $n \in \mathbb{N}$. By [B-GT] II $\S 3.5$ Thm. 1 it suffices to show that, for any $m>n$, the image of $M_{m}$ in $M_{n}$ is dense. Choose an $A_{q_{m}}$-linear surjection $A_{q_{m}}^{r} \longrightarrow M_{m}$ from a finitely generated free $A_{q_{m}}$-module onto $M_{m}$. Then all horizontal arrows in the commutative diagram

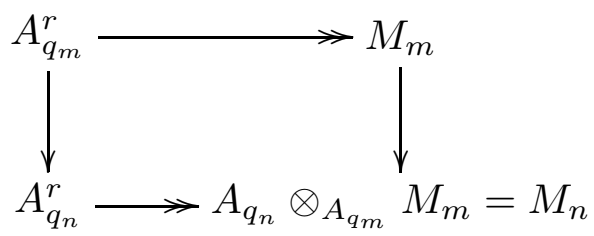

are surjective. Our claim now follows from the fact that the left vertical arrow has dense image and that, by Prop. 2.1.iii, all the maps in the diagram are continuous for the canonical topologies.

ii. The system $\left(M_{n}\right)_{n}$, by Theorem A, has the Mittag-Leffler property as formulated in [EGA] III 0.13.2.4 so that loc. cit. 13.2.2 applies.

It is immediate from Theorem $\mathrm{B}$ that the functor $\Gamma: \operatorname{Coh}_{\left(A,\left(q_{n}\right)\right)} \longrightarrow \operatorname{Mod}(A)$ is exact.

Corollary 3.1: For any coherent sheaf $\left(M_{n}\right)_{n}$ for $\left(A,\left(q_{n}\right)\right)$ and $M:=\Gamma\left(M_{n}\right)$ the natural map

$$
A_{q_{n}} \otimes_{A} M \stackrel{\cong}{\longrightarrow} M_{n}
$$

is an isomorphism for any $n \in \mathbb{N}$.

Proof: By Theorem A the $A_{q_{n}}$-submodule of $M_{n}$ generated by the image of $M$ is dense in $M_{n}$. Prop. 2.1.ii. then says that this submodule, in fact, must be equal to $M_{n}$. This establishes the surjectivity of the map in question and, more precisely, that $M_{n}$ as an $A_{q_{n}}$-module is generated by finitely many elements in the image of the map $M \longrightarrow M_{n}$. Suppose now that $b_{1} \otimes x_{1}+\ldots+b_{k} \otimes x_{k} \in$ $A_{q_{n}} \otimes_{A} M$ is an element such that $b_{1} x_{1}+\ldots+b_{k} x_{k}=0$ in $M_{n}$. Consider the homomorphism of coherent sheaves

$$
\begin{aligned}
\left(A_{q_{n^{\prime}}}^{k}\right)_{n^{\prime}} & \longrightarrow\left(M_{n^{\prime}}\right)_{n^{\prime}} \\
\left(a_{1}, \ldots, a_{k}\right) & \longmapsto a_{1} x_{1}+\ldots a_{k} x_{k} .
\end{aligned}
$$


Applying the above surjectivity argument to its kernel we find finitely many elements $\left(c_{1}^{(1)}, \ldots, c_{k}^{(1)}\right), \ldots,\left(c_{1}^{(r)}, \ldots, c_{k}^{(r)}\right)$ in $A^{k}$ whose images generate the kernel of the map $A_{q_{n}}^{k} \longrightarrow M_{n}$ as an $A_{q_{n}}$-module. In particular there are $f_{1}, \ldots, f_{r} \in$ $A_{q_{n}}$ such that

$$
\left(b_{1}, \ldots, b_{k}\right)=f_{1} \cdot\left(c_{1}^{(1)}, \ldots, c_{k}^{(1)}\right)+\ldots+f_{r} \cdot\left(c_{1}^{(r)}, \ldots, c_{k}^{(r)}\right) .
$$

It follows that

$$
\begin{aligned}
\sum_{i=1}^{k} b_{i} \otimes x_{i} & =\sum_{i=1}^{k} \sum_{j=1}^{r} f_{j} c_{i}^{(j)} \otimes x_{i}=\sum_{i=1}^{k} \sum_{j=1}^{r} f_{j} \otimes c_{i}^{(j)} x_{i} \\
& =\sum_{j=1}^{r} f_{j} \otimes\left(\sum_{i=1}^{k} c_{i}^{(j)} x_{i}\right)=0 .
\end{aligned}
$$

Hence the map in question is injective as well.

Remark 3.2: $A_{q_{n}}$, for any $n \in \mathbb{N}$, is flat as a right $A$-module.

Proof: It suffices to show that for any left ideal $L \subseteq A$ the induced map $A_{q_{n}} \otimes_{A}$ $L \longrightarrow A_{q_{n}}$ is injective. But this is exactly the same computation as in the previous proof.

Corollary 3.3: The functor $\Gamma: \operatorname{Coh}_{\left(A,\left(q_{n}\right)\right)} \stackrel{\sim}{\longrightarrow} \mathcal{C}_{A}$ is an equivalence of categories.

Proof: By definition the functor is essentially surjective. According to the previous corollary it is fully faithful. Both properties together amount to the functor being an equivalence of categories.

Corollary 3.4: $i$. The direct sum of two coadmissible A-modules is coadmissible;

ii. the (co)kernel and (co)image of an arbitary A-linear map between coadmissible A-modules are coadmissible;

iii. the sum of two coadmissible submodules of a coadmissible A-module is coadmissible;

iv. any finitely generated submodule of a coadmissible A-module is coadmissible;

$v$. any finitely presented A-module is coadmissible.

Proof: The first assertion is obvious. The last three assertions are immediate consequences of the first two. Hence it remains to establish the second assertion. By the previous corollary any map between coadmissible modules comes from a map between coherent sheaves. But, by Theorem $\mathrm{B}$, the functor $\Gamma$ into $\operatorname{Mod}(A)$ commutes with the formation of (co)kernels and (co)images. 
Corollary 3.5: $\mathcal{C}_{A}$ is an abelian subcategory of $\operatorname{Mod}(A)$.

We want to discuss a few permanence properties of our notions. First of all the subcategory $\mathcal{C}_{A}$ in $\operatorname{Mod}(A)$ is not closed with respect to the passage to submodules or quotient modules. To obtain a precise statement we will equip any coadmissible $A$-module $M$ with a topology. Write

$$
M=\lim _{n} M_{n}
$$

By Prop. 2.1.i, each $M_{n}$ carries its canonical Banach space topology as a finitely generated $A_{q_{n}}$-module. We equip $M$ with the projective limit topology of these canonical topologies. This makes $M$ into a $K$-Fréchet space. Moreover, the $A$-module structure map $A \times M \rightarrow M$ clearly is continuous. We will call this Fréchet topology the canonical topology of $M$.

Lemma 3.6: For any coadmissible A-module $M$ and any submodule $N \subseteq M$ the following assertions are equivalent:

i. $N$ is coadmissible;

ii. $M / N$ is coadmissible;

iii. $N$ is closed in the canonical topology of $M$.

Proof: The equivalence of i. and ii. is immediate from Cor. 3.4.ii. Suppose that $M=\Gamma\left(M_{n}\right)$. For any $n \in \mathbb{N}$ let $N_{n} \subseteq M_{n}$ denote the $A_{q_{n}}$-submodule generated by the image of $N$. Then $\left(N_{n}\right)_{n}$ is a coherent subsheaf of $\left(M_{n}\right)_{n}$. Moreover, it is straightforward to see that $\bar{N}:=\Gamma\left(N_{n}\right)$ is the closure of $N$ in $M$ with its canonical topology. Hence if $N$ is closed then it is coadmissible. On the other hand, if $N$ is coadmissible then Cor. 3.1 implies that $N=\Gamma\left(N_{n}\right)=\bar{N}$.

It is immediate from Prop. 2.1.iii and Cor. 3.3 that any $A$-linear map $f$ : $M \longrightarrow N$ between two coadmissible $A$-modules is continuous for the canonical topologies. It also is strict since its image is a coadmissible submodule of $N$ by Cor. 3.4.ii and hence is closed in $N$ by Lemma 3.6; so $f: M \longrightarrow \operatorname{im}(f)$ is a continuous surjection between Fréchet spaces which by the open mapping theorem is strict.

As another consequence of Cor. 3.4.iv and Lemma 3.6 we obtain that any finitely generated left ideal of $A$ is closed.

Proposition 3.7: Let I be a closed two sided ideal in a K-Fréchet-Stein algebra $A$; then $A / I$ is a $K$-Fréchet-Stein algebra as well. 
Proof: By Lemma 3.6 both, $I$ and $A / I$, are coadmissible $A$-modules. We therefore have the exact sequences

$$
0 \longrightarrow A_{q_{n}} \otimes_{A} I \longrightarrow A_{q_{n}} \longrightarrow A_{q_{n}} / A_{q_{n}} I \longrightarrow 0
$$

and the isomorphism of $A$-modules

$$
A / I \stackrel{\cong}{\underset{n}{\longrightarrow}} \lim _{q_{n}} A_{q_{n}} / A_{q_{n}} I .
$$

Moreover $A_{q_{n}} I$ is the closure of the image of $I$ in $A_{q_{n}}$. The latter implies that $A_{q_{n}} I$ is a two sided ideal in $A_{q_{n}}$. Hence $A_{q_{n}} / A_{q_{n}} I$ is a $K$-Banach algebra with respect to the quotient norm $\overline{q_{n}}$ of $q_{n}$. As a consequence of the open mapping theorem $(*)$ then is an isomorphism of $K$-Fréchet algebras. This means that $\overline{q_{1}} \leq \ldots \leq \overline{q_{n}} \leq \ldots$ is a sequence of algebra seminorms on $A / I$ which define the quotient topology. As a quotient of a noetherian algebra $A_{q_{n}} / A_{q_{n}} I=(A / I)_{\overline{q_{n}}}$ is noetherian as well. By construction we have

$$
\begin{aligned}
(A / I)_{\overline{q_{n}}} & =A_{q_{n}} \otimes_{A}(A / I)=A_{q_{n}} \otimes_{A_{q_{n+1}}}\left(A_{q_{n+1}} \otimes_{A}(A / I)\right) \\
& =A_{q_{n}} \otimes_{A_{q_{n+1}}}(A / I)_{\overline{q_{n+1}}}
\end{aligned}
$$

as $\left(A_{q_{n}},(A / I)_{\overline{q_{n+1}}}\right)$-bimodules. The flatness of $A_{q_{n}}$ as a right $A_{q_{n+1}}$-module therefore implies, by base change (compare [B-CA] I $\S 2.7$ Cor. 2), that $(A / I)_{\overline{q_{n}}}$ is flat as a right $(A / I)_{\overline{q_{n+1}}}$-module.

Lemma 3.8: Let $A \longrightarrow B$ be a continuous unital algebra homomorphism between $K$-Fréchet-Stein algebras such that $B$ is coadmissible as a (left) $A$-module, and let $M$ be a (left) $B$-module; then $M$ is coadmissible as a B-module if and only if it is coadmissible as an A-module.

Proof: Let $\left(q_{n}\right)_{n}$, resp. $\left(p_{n}\right)_{n}$, be a sequence of seminorms on $A$, resp. $B$, as required in the definition of a Fréchet-Stein algebra. Since the homomorphism $A \longrightarrow B$ is continuous we find, for any $n \in \mathbb{N}$, an $m \geq n$ such that this map extends continuously to a homomorphism of Banach algebras $A_{q_{m}} \longrightarrow B_{p_{n}}$. By passing to a subsequence of $\left(q_{n}\right)_{n}$ (and renumbering) we may assume that we always can choose $m=n$. We then have, for any $n \in \mathbb{N}$, the commutative diagram

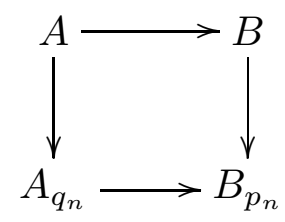

and hence the induced $\left(A_{q_{n}}, B\right)$-bimodule map

$$
A_{q_{n}} \otimes_{A} B \longrightarrow B_{p_{n}}
$$


which is continuous for the canonical topology on the finitely generated $A_{q_{n}}$ module $A_{q_{n}} \otimes_{A} B$. In the limit with respect to $n$ these maps give back the identity map on $B$, but as a continuous map from $B$ with its canonical topology as a coadmissible $A$-module into $B$ with its given Fréchet topology. By the open mapping theorem these two topologies then must in fact coincide. Hence, for each $n \in \mathbb{N}$, the canonical $(A, B)$-bimodule map $B \longrightarrow A_{q_{n}} \otimes_{A} B$ is continuous and factorizes therefore through a continuous $\left(A_{q_{m(n)}}, B\right)$-bimodule map $B_{p_{m(n)}} \longrightarrow A_{q_{n}} \otimes_{A} B$ for some $m(n) \geq n$. This proves that we have

$$
\lim _{n} B_{p_{n}} \otimes_{B} M=\lim _{\lim _{n}}\left(A_{q_{n}} \otimes_{A} B\right) \otimes_{B} M=\underset{\lim _{n}}{A_{q_{n}}} \otimes_{A} M
$$

If $A_{q_{n}} \otimes_{A} M$ is a finitely generated $A_{q_{n}}$-module then $B_{p_{n}} \otimes_{A_{q_{n}}}\left(A_{q_{n}} \otimes_{A} M\right)=$ $B_{p_{n}} \otimes_{A} M$ and hence its quotient $B_{p_{n}} \otimes_{B} M$ are finitely generated $B_{p_{n}}$-modules. Let us assume, vice versa, that $B_{p_{m(n)}} \otimes_{B} M$ is a finitely generated $B_{p_{m(n)}}{ }^{-}$ module. We choose a set of generators $1 \otimes x_{1}, \ldots, 1 \otimes x_{k}$ with $x_{i} \in M$. We also fix generators $1 \otimes y_{1}, \ldots, 1 \otimes y_{l}$, with $y_{j} \in B$, of the finitely generated $A_{q_{n}}$-module $A_{q_{n}} \otimes_{A} B$. Given any $x \in M$ we find $b_{i} \in B_{p_{m(n)}}$ such that

$$
1 \otimes x=\sum_{i=1}^{k} b_{i} \otimes x_{i}
$$

in $B_{p_{m(n)}} \otimes_{B} M$. For each $b_{i}$ we then find $c_{i, j} \in A_{q_{n}}$ such that

$$
\sum_{j=1}^{l} c_{i, j} \otimes y_{j}=\text { image of } b_{i} \text { in } A_{q_{n}} \otimes_{A} B
$$

By first applying the map $B_{p_{m(n)}} \otimes_{B} M \longrightarrow\left(A_{q_{n}} \otimes_{A} B\right) \otimes_{B} M=A_{q_{n}} \otimes_{A} M$ and then inserting (2) the identity (1) becomes

$$
1 \otimes 1 \otimes x=\sum_{i, j} c_{i, j} \otimes y_{j} \otimes x_{i}, \quad \text { resp. } 1 \otimes x=\sum_{i, j} c_{i, j} \otimes y_{j} x_{i} .
$$

This proves that the finitely many elements $1 \otimes y_{j} x_{i}$ generate $A_{q_{n}} \otimes_{A} M$ as an $A_{q_{n}}$-module.

In the setting of Prop. 3.7 this last lemma means that

$$
\mathcal{C}_{A / I}=\operatorname{Mod}(A / I) \cap \mathcal{C}_{A}
$$

We finish this section with a general simplicity criterion for coadmissible modules. 
Lemma 3.9: Let $M$ be a coadmissible A-module corresponding to the coherent sheaf $\left(M_{n}\right)_{n}$; the $A$-module $M$ is simple if the $A_{q_{n}}$-module $M_{n}$ is simple for infinitely many $n \in \mathbb{N}$.

Proof: Suppose $M$ is not simple and is nonzero. We then find a nonzero finitely generated proper $A$-submodule $0 \neq N \subset M$. By Cor. 3.4.iv $N$ is coadmissible and therefore corresponds to a nonzero proper subsheaf $0 \neq\left(N_{n}\right)_{n} \subset\left(M_{n}\right)_{n}$. There have to exist $n_{0}, n_{1} \in \mathbb{N}$ such that $N_{n_{0}} \neq 0$ and $N_{n_{1}} \neq M_{n_{1}}$. As a consequence of Cor. 3.1 any $n \geq \sup \left(n_{0}, n_{1}\right)$ then satisfies $N_{n} \neq 0$ and $N_{n} \neq M_{n}$. In other words, for these $n$ the $A_{q_{n}}$-module $M_{n}$ is not simple.

\section{Distribution algebras of uniform pro-p-groups}

Let $\mathbb{Q}_{p} \subseteq L \subseteq K \subseteq \mathbb{C}_{p}$ be complete intermediate fields where $L / \mathbb{Q}_{p}$ is finite and $K$ is discretely valued. The absolute value || on $\mathbb{C}_{p}$ is normalized as usual by $|p|=p^{-1}$. Furthermore, let $G$ be a compact locally $L$-analytic group. In [ST2] 2 we have introduced the $K$-Fréchet algebra $D(G, K)$ of $K$-valued locally analytic distributions on $G$. One of the principal aims of this paper is to show that $D(G, K)$ has a natural structure of a $K$-Fréchet-Stein algebra. The crucial case to consider is $L=\mathbb{Q}_{p}$ and $G$ a locally $\mathbb{Q}_{p}$-analytic group which in addition is a uniform pro- $p$-group. We remark right away that any compact locally $\mathbb{Q}_{p^{-}}$ analytic group has an open normal subgroup which is a uniform pro-p-group (compare [DDMS] Cor. 8.34). The technical heart of the matter is the use and appropriate generalization of the methods of Lazard in [Laz]. It therefore seems quite natural and, in fact, allows for more flexibility to also use his language. So we begin by reviewing some of his basic notions and results.

A $p$-valuation on a group $G$ is a real valued function $\omega: G \backslash\{1\} \longrightarrow(1 /(p-1), \infty)$ such that

$$
\begin{aligned}
& \omega\left(g h^{-1}\right) \geq \min (\omega(g), \omega(h)), \\
& \omega\left(g^{-1} h^{-1} g h\right) \geq \omega(g)+\omega(h), \text { and } \\
& \omega\left(g^{p}\right)=\omega(g)+1
\end{aligned}
$$

for any $g, h \in G$ ([Laz] III.2.1.2). As usual one puts $\omega(1):=\infty$. For each real number $\nu>0$ we define the normal subgroups

$$
G_{\nu}:=\{g \in G: \omega(g) \geq \nu\} \quad \text { and } \quad G_{\nu^{+}}:=\{g \in G: \omega(g)>\nu\}
$$

of $G$, and we put

$$
g r(G):=\bigoplus_{\nu>0} G_{\nu} / G_{\nu^{+}} .
$$

With the Lie bracket induced by the commutator $\operatorname{gr}(G)$ is a graded Lie algebra over $\mathbb{F}_{p}$. Let $\mathbb{F}_{p}[\epsilon]$ denote the polynomial ring in one variable $\epsilon$ over $\mathbb{F}_{p}$, as usual viewed as a graded $\mathbb{F}_{p^{-}}$algebra with $\epsilon$ of degree 1 . The rule $g G_{\nu^{+}} \mapsto g^{p} G_{(\nu+1)^{+}}$ defines an $\mathbb{F}_{p}$-linear operator $P$ on $\operatorname{gr}(G)$, which is homogeneous of degree 1 , 
and which satisfies $[P \bar{g}, \bar{h}]=P([\bar{g}, \bar{h}])$ for homogeneous elements $\bar{g}, \bar{h}$ of $\operatorname{gr}(G)$. Letting $\epsilon$ act as $P$ therefore makes $g r(G)$ into a graded Lie algebra over $\mathbb{F}_{p}[\epsilon]$ ([Laz] III.2.1.1). As an $\mathbb{F}_{p}[\epsilon]$-module $\operatorname{gr}(G)$ is free and its rank is called the rank of $G$ ([Laz $]$ III.2.1.3).

For the remainder of this section we fix a compact locally $\mathbb{Q}_{p}$-analytic group $G$ together with a $p$-valuation $\omega$ on $G$. As a consequence of [Laz] III. 3.1.3/7/9 we have:

- The topology of $G$ is defined by $\omega$ ([Laz] II.1.1.5); in particular $G$ is a pro- $p$ group.

- The rank of $G$ is equal to the dimension $d$ of $G$ (as a locally analytic manifold) and in particular is finite.

By [Laz] III.3.1.11 a given $p$-valuation on $G$ can always be changed into one which has rational values. We therefore assume from now on that $\omega$ has rational values.

An ordered basis of $G$ is a sequence of elements $h_{1}, \ldots, h_{d} \in G \backslash\{1\}$ such that the elements $h_{i} G_{\omega\left(h_{i}\right)}+\in \operatorname{gr}(G)$ form a basis of $g r(G)$ as an $\mathbb{F}_{p}[\epsilon]$-module. It always exists. Given one the map

$$
\begin{array}{rccc}
\psi: & \mathbb{Z}_{p}^{d} & \stackrel{\sim}{\longrightarrow} & G \\
\left(x_{1}, \ldots x_{d}\right) & \longmapsto & h_{1}^{x_{1}} \cdot \ldots \cdot h_{d}^{x_{d}}
\end{array}
$$

is a bijective global chart for the manifold $G$ satisfying

$$
\omega\left(h_{1}^{x_{1}} \cdot \ldots \cdot h_{d}^{x_{d}}\right)=\min _{1 \leq i \leq d}\left(\omega\left(h_{i}\right)+\omega_{p}\left(x_{i}\right)\right)
$$

where $\omega_{p}$ denotes the $p$-adic valuation on $\mathbb{Z}_{p}$ ([Laz $]$ III.2.2.5/6). In the following we fix an ordered basis $\left(h_{1}, \ldots, h_{d}\right)$ of $G$ together with the corresponding chart $\psi$. Using this chart we may identify the locally convex $K$-vector spaces of locally analytic functions

$$
\psi^{*}: C^{a n}(G, K) \stackrel{\cong}{\longrightarrow} C^{a n}\left(\mathbb{Z}_{p}^{d}, K\right)
$$

as well as the larger $K$-Banach spaces of continuous functions

$$
\psi^{*}: C(G, K) \stackrel{\cong}{\longrightarrow} C\left(\mathbb{Z}_{p}^{d}, K\right)
$$

on both sides. Via Mahler expansions (compare [Laz] III.1.2.4) $C\left(\mathbb{Z}_{p}^{d}, K\right)$ can be viewed as the space of all series

$$
f(x)=\sum_{\alpha \in \mathbb{N}_{0}^{d}} c_{\alpha}\left(\begin{array}{l}
x \\
\alpha
\end{array}\right)
$$


with $c_{\alpha} \in K$ and such that $\left|c_{\alpha}\right| \longrightarrow 0$ as $|\alpha| \longrightarrow \infty$. Here we put, as usual,

$$
\left(\begin{array}{l}
x \\
\alpha
\end{array}\right):=\left(\begin{array}{l}
x_{1} \\
\alpha_{1}
\end{array}\right) \cdots\left(\begin{array}{l}
x_{d} \\
\alpha_{d}
\end{array}\right)
$$

and

$$
|\alpha|:=\sum_{i=1}^{d} \alpha_{i}
$$

for $x=\left(x_{1}, \ldots, x_{d}\right) \in \mathbb{Z}_{p}^{d}$ and multi-indices $\alpha=\left(\alpha_{1}, \ldots, \alpha_{d}\right) \in \mathbb{N}_{0}^{d}$. By Amice's theorem (compare [Laz] III.1.3.9) the Mahler expansion $f$ lies in the subspace $C^{a n}\left(\mathbb{Z}_{p}^{d}, K\right)$ if and only if $\left|c_{\alpha}\right| r^{|\alpha|} \longrightarrow 0$ for some real number $r>1$ as $|\alpha| \longrightarrow \infty$.

We embed the group ring $\mathbb{Z}_{p}[G]$ into the distribution algebra $D(G, K)$ by viewing a group element $g \in G$ as the Dirac distribution $\delta_{g}$. We then have

$$
g(f)=\delta_{\psi(x)}(f)=\psi^{*}(f)(x)
$$

for any $f \in C(G, K)$ and any $g=\psi(x) \in G$. Henceforth we write $b_{i}:=h_{i}-1$ and $\mathbf{b}^{\alpha}:=b_{1}^{\alpha_{1}} b_{2}^{\alpha_{2}} \cdots b_{d}^{\alpha_{d}}$, for $\alpha=\left(\alpha_{1}, \ldots, \alpha_{d}\right) \in \mathbb{N}_{0}^{d}$, in $\mathbb{Z}_{p}[G] \subseteq D(G, K)$. If $c_{\alpha}$ denote the coefficients of the Mahler expansion of $\psi^{*}(f)$ for some $f \in C(G, K)$ then their classical computation in terms of finite differences shows that

$$
\mathbf{b}^{\alpha}(f)=c_{\alpha}
$$

It easily follows that any distribution $\lambda \in D(G, K)$ has a unique convergent expansion

$$
\lambda=\sum_{\alpha \in \mathbb{N}_{0}^{d}} d_{\alpha} \mathbf{b}^{\alpha}
$$

with $d_{\alpha} \in K$ such that, for any $0<r<1$, the set $\left\{\left|d_{\alpha}\right| r^{|\alpha|}\right\}_{\alpha \in \mathbb{N}_{0}^{d}}$ is bounded. Conversely, any such series is convergent in $D(G, K)$. The Fréchet topology on $D(G, K)$ is defined by the family of norms

$$
\|\lambda\|_{r}^{\prime}:=\sup _{\alpha \in \mathbb{N}_{0}^{d}}\left|d_{\alpha}\right| r^{|\alpha|}
$$

for $0<r<1$. For our purposes it is in fact more convenient to take the original $p$-valuation $\omega$ into account and to always work with the equivalent set of norms

$$
\|\lambda\|_{r}:=\sup _{\alpha \in \mathbb{N}_{0}^{d}}\left|d_{\alpha}\right| r^{\tau \alpha}
$$


where $\tau \alpha:=\sum_{i} \alpha_{i} \omega\left(h_{i}\right)$. We note that, since the multiplication in $D(G, K)$ is jointly continuous, we obtain the expansion of the product of two distributions by multiplying their expansions, inserting the expansions

$$
\mathbf{b}^{\beta} \mathbf{b}^{\gamma}=\sum_{\alpha} c_{\beta \gamma, \alpha} \mathbf{b}^{\alpha}
$$

and rearranging.

The inclusion $\mathbb{Z}_{p}[G] \subseteq D(G, K)$ extends to an embedding of topological rings

$$
\mathbb{Z}_{p}[[G]] \hookrightarrow D(G, K)
$$

of the completed group ring

$$
\mathbb{Z}_{p}[[G]]:=\lim _{\longleftarrow} \mathbb{Z}_{p}[G / N]
$$

with $N$ running over all open normal subgroups of $G$ into the distribution algebra. A distribution $\lambda=\sum_{\alpha} d_{\alpha} \mathbf{b}^{\alpha}$ lies in $\mathbb{Z}_{p}[[G]]$ if and only if all $d_{\alpha} \in \mathbb{Z}_{p}$; moreover, the norm \|\|$_{1 / p}$ restricted to $\mathbb{Z}_{p}[[G]]$ is independent of the choice of the ordered basis of $G$, is multiplicative, satisfies $\|g-1\|_{1 / p} \leq p^{-\omega(g)}$ for any $g \in G$, and defines the compact topology of $\mathbb{Z}_{p}[[G]]$ ([Laz] III.2.3; his $w$ is the additive version of our $\left.\|_{1 / p}\right)$. The latter in fact implies that each of the norms \|\|$_{r}$, for $0<r<1$, defines this same compact topology of $\mathbb{Z}_{p}[[G]]$. Theorem III.2.3.3 in [Laz $]$ says that the inclusion map $G \hookrightarrow \mathbb{Z}_{p}[[G]]$ induces an isomorphism

$$
U(\operatorname{gr}(G)) \stackrel{\cong}{\longrightarrow} g r_{1 / p} \mathbb{Z}_{p}[[G]]
$$

where the left hand side is the universal enveloping algebra of the Lie algebra $\operatorname{gr}(G)$ over $\mathbb{F}_{p}[\epsilon]$ and the right hand side is the associated graded ring for the filtration

$$
F_{1 / p}^{s} \mathbb{Z}_{p}[[G]]:=\left\{\lambda \in \mathbb{Z}_{p}[[G]]:\|\lambda\|_{1 / p} \leq p^{-s}\right\}
$$

Note that the right hand side is naturally an $\mathbb{F}_{p}[\epsilon]$-algebra as well by viewing $\mathbb{F}_{p}[\epsilon]$ as the associated graded ring for the $p$-adic valuation on $\mathbb{Z}_{p}$.

After this review we begin our investigation by looking at the expansions

$$
\mathbf{b}^{\beta} \mathbf{b}^{\gamma}=\sum_{\alpha} c_{\beta \gamma, \alpha} \mathbf{b}^{\alpha}
$$

with $c_{\beta \gamma, \alpha} \in \mathbb{Z}_{p}$

Lemma 4.1: i. $\omega_{p}\left(c_{\beta \gamma, \alpha}\right) \geq \max (0, \tau \beta+\tau \gamma-\tau \alpha)$;

ii. $\left|c_{\beta \gamma, \alpha}\right| r^{\tau \alpha} \leq r^{\tau \beta+\tau \gamma}$ for any $1 / p \leq r \leq 1$. 
Proof: Since \|\|$_{1 / p}$ is multiplicative on $\mathbb{Z}_{p}[[G]]$ we have

$$
p^{-\tau \beta-\tau \gamma}=\left\|\mathbf{b}^{\beta} \mathbf{b}^{\gamma}\right\|_{1 / p}=\sup _{\alpha}\left|c_{\beta \gamma, \alpha}\right| p^{-\tau \alpha}
$$

which implies $\omega_{p}\left(c_{\beta \gamma, \alpha}\right) \geq \tau \beta+\tau \gamma-\tau \alpha$. It also implies the second assertion in case $r=1 / p$. For general $r$ we distinguish two cases. If $\tau \alpha \geq \tau \beta+\tau \gamma$ then $\left|c_{\beta \gamma, \alpha}\right| \leq 1 \leq r^{\tau \beta+\tau \gamma-\tau \alpha}$. If $\tau \alpha \leq \tau \beta+\tau \gamma$ then $\left|c_{\beta \gamma, \alpha}\right| \leq(1 / p)^{\tau \beta+\tau \gamma-\tau \alpha} \leq$ $r^{\tau \beta+\tau \gamma-\tau \alpha}$.

Proposition 4.2: Each norm \|\|$_{r}$ on $D(G, K)$, for $1 / p \leq r<1$, is submultiplicative.

Proof: Let $\lambda=\sum_{\beta} d_{\beta} \mathbf{b}^{\beta}$ and $\mu=\sum_{\gamma} e_{\gamma} \mathbf{b}^{\gamma}$ be two distributions in $D(G, K)$. Their product then has the expansion

$$
\lambda \mu=\sum_{\beta, \gamma} d_{\beta} e_{\gamma} \mathbf{b}^{\beta} \mathbf{b}^{\gamma}=\sum_{\alpha}\left(\sum_{\beta, \gamma} d_{\beta} e_{\gamma} c_{\beta \gamma, \alpha}\right) \mathbf{b}^{\alpha}
$$

Using Lemma 4.1.ii we obtain

$$
\begin{aligned}
\|\lambda \mu\|_{r} & =\sup _{\alpha}\left|\sum_{\beta, \gamma} d_{\beta} e_{\gamma} c_{\beta \gamma, \alpha}\right| r^{\tau \alpha} \leq \sup _{\alpha, \beta, \gamma}\left|d_{\beta}\right|\left|e_{\gamma}\right|\left|c_{\beta \gamma, \alpha}\right| r^{\tau \alpha} \\
& \leq \sup _{\beta, \gamma}\left|d_{\beta}\right| r^{\tau \beta}\left|e_{\gamma}\right| r^{\tau \gamma} \\
& \leq\|\lambda\|_{r}\|\mu\|_{r} .
\end{aligned}
$$

We put

$$
K[[G]]:=K \otimes_{\mathbb{Q}_{p}} \mathbb{Z}_{p}[[G]]
$$

which still is a subalgebra of $D(G, K)$. On any of the rings $R=\mathbb{Z}_{p}[[G]], K[[G]]$, or $D(G, K)$ we have, for each $1 / p \leq r<1$, the filtration

$$
F_{r}^{s} R:=\left\{a \in R:\|a\|_{r} \leq p^{-s}\right\}
$$

The associated graded ring is denoted by $g r_{r}^{\cdot} R$. We let

$$
D_{r}(G, K):=\text { completion of } D(G, K) \text { with respect to the norm }\|\|_{r} \text {. }
$$

As a $K$-Banach space $D_{r}(G, K)$ is given by all series

$$
\sum_{\alpha} d_{\alpha} \mathbf{b}^{\alpha} \text { with } d_{\alpha} \in K \text { and }\left|d_{\alpha}\right| r^{\tau \alpha} \longrightarrow 0 \text { as }|\alpha| \longrightarrow \infty
$$


We also introduce the even larger $K$-Banach space

$$
D_{<r}(G, K):=\left\{\sum_{\alpha} d_{\alpha} \mathbf{b}^{\alpha}: d_{\alpha} \in K \text { and }\left\{\left|d_{\alpha}\right| r^{\tau \alpha}\right\}_{\alpha} \text { is bounded }\right\}
$$

On both of them the norm continues to be given by

$$
\left\|\sum_{\alpha} d_{\alpha} \mathbf{b}^{\alpha}\right\|_{r}=\sup _{\alpha}\left|d_{\alpha}\right| r^{\tau \alpha}
$$

By Prop. 4.2 the multiplication on $D(G, K)$ extends continuously to $D_{r}(G, K)$ and makes $D_{r}(G, K)$ into a $K$-Banach algebra. If $r>1 / p$ then $D_{<r}(G, K) \subseteq$ $D_{1 / p}(G, K)$. The computation in the proof of Prop. 4.2 shows that the left term is multiplicatively closed in the algebra $D_{1 / p}(G, K)$ and that $D_{<r}(G, K)$ with the norm \|\|$_{r}$ is a $K$-Banach algebra. Altogether we obtain the system of $K$-Banach algebras

$$
\ldots \subseteq D_{r}(G, K) \subseteq D_{<r}(G, K) \subseteq D_{r^{\prime}}(G, K) \subseteq \ldots \subseteq D_{1 / p}(G, K)
$$

with $1 / p \leq r^{\prime}<r<1$ and

$$
D(G, K)={\underset{\lim }{r}}_{r} D_{r}(G, K)={\underset{\lim }{r}}_{r} D_{<r}(G, K)
$$

On $R=D_{r}(G, K)$, resp. $=D_{<r}(G, K)$, we again have, for any $1 / p \leq r^{\prime} \leq r$, resp. $1 / p \leq r^{\prime}<r$, the filtration

$$
F_{r^{\prime}}^{s} R:=\left\{a \in R:\|a\|_{r^{\prime}} \leq p^{-s}\right\}
$$

with associated graded ring $g r_{r^{\prime}}^{\cdot} R$.

It is crucial for our results that all the various graded rings we have introduced can be computed explicitly. First of all we remark that all the norms under consideration restrict on $\mathbb{Z}_{p}$ to the $p$-adic absolute value. Hence all graded rings naturally are $\mathbb{F}_{p}[\epsilon]$-algebras. Secondly we make the observation that, since $K[[G]]$ is dense in $D_{r}(G, K)$, we have the isomorphism

$$
g r_{r} K\left[[G] \stackrel{\cong}{\longrightarrow} g r_{r} D_{r}(G, K)\right.
$$

for any $1 / p \leq r<1$. Moreover, by construction, the natural map of $\mathbb{F}_{p}[\epsilon]$ algebras $g r_{r} \mathbb{Z}_{p}[[G]] \hookrightarrow g r_{r} K[[G]]$ is injective. It extends to a ring homomorphism

$$
g r \cdot K \underset{\mathbb{F}_{p}[\epsilon]}{\otimes} g r_{r}^{\cdot} \mathbb{Z}_{p}[[G]] \longrightarrow g r_{r}^{\cdot} K[[G]]
$$


where $\mathrm{gr}^{\cdot} K$ is the associated graded ring for the filtration $F^{s} K:=\{a \in K:|a| \leq$ $\left.p^{-s}\right\}$. This map, in fact, is an isomorphism since $g r_{r} \mathbb{Z}_{p}[[G]]$, resp. $g r_{r} K[[G]]$, is free as a module over $\mathbb{F}_{p}[\epsilon]$, resp. $g r^{\cdot} K$, with basis the principal symbols $\sigma_{r}\left(\mathbf{b}^{\alpha}\right)$. We recall that the principal symbol $\sigma_{r}(a)$ of a nonzero element $a$ in one of our rings $R$ with respect to the filtration $F_{r}^{\cdot} R$ is the coset $\sigma_{r}(a):=a+F_{r}^{s+} R$ if $a \in F_{r}^{s} R \backslash F_{r}^{s+} R$.

Lemma 4.3: For any $1 / p \leq r<1$ we have

$$
g r \cdot K \underset{\mathbb{F}_{p}[\epsilon]}{\otimes} g r_{r} \mathbb{Z}_{p}[[G]] \stackrel{\cong}{\longrightarrow} g r_{r} K[[G]] \stackrel{\cong}{\longrightarrow} g r_{r} D_{r}(G, K)
$$

We note that

$$
g r \cdot K \cong k\left[\epsilon_{\mathrm{o}}, \epsilon_{\mathrm{o}}^{-1}\right]
$$

where $k$ is the residue class field of $K$ and $\epsilon_{\mathrm{o}}$ denotes the principal symbol of a prime element of $K$. The natural map $\mathbb{F}_{p}[\epsilon] \hookrightarrow g r^{\cdot} K$ is given by $\epsilon \mapsto a \epsilon_{\mathrm{o}}^{e}$ where $e$ denotes the absolute ramification index of $K$ and $a \in \mathbb{F}_{p}^{\times}$is an appropriate element.

To obtain our results we have to impose additional condition on our $p$-valued group $(G, \omega)$. According to [Laz] III.2.1.6 the pair $(G, \omega)$ is called $p$-saturated if any $g \in G$ such that $\omega(g)>p /(p-1)$ is a $p^{\text {th }}$ power. In the following we make the assumption that

$$
\begin{aligned}
& (G, \omega) \text { is } p \text {-saturated and the ordered basis }\left(h_{1}, \ldots, h_{d}\right) \text { of } G \\
& \text { satisfies } \omega\left(h_{i}\right)+\omega\left(h_{j}\right)>p /(p-1) \text { for any } 1 \leq i \neq j \leq d .
\end{aligned}
$$

Remark: In [DDMS] $\S 4.1$ the authors introduce the class of uniform (or uniformly powerful) pro-p-groups. Without recalling their definition we briefly relate this notion to the language of Lazard which we are using. For simplicity we assume that $p \neq 2$. First let $G$ be, as above, a compact locally $\mathbb{Q}_{p}$-analytic group which has a $p$-valuation $\omega$ such that (HYP) holds. As we have seen in the proof of Lemma 4.4 the commutator of any two members of the given ordered basis is a $p$-th power. This easily implies that $G$ is powerful ([DDMS] Def. 3.1). On the other hand any group with a $p$-valuation must be torsionfree. According to [DDMS] Thm. 4.5 these two properties together ensure that $G$ is uniform.

Vice versa, assume $G$ to be a uniform pro-p-group. It is locally $\mathbb{Q}_{p}$-analytic by [DDMS] Thm. 8.18. The discussion in [DDMS] $\S 4.2$ in fact shows that $G$ has an integrally valued $p$-valuation $\omega^{\prime}$ and an ordered basis $\left(h_{1}, \ldots, h_{d}\right)$ such that $\omega^{\prime}\left(h_{1}\right)=\ldots=\omega^{\prime}\left(h_{d}\right)=1$; moreover, by [DDMS] Lemma 4.10, each element in $G_{2}$ is a $p$-th power. (Compare also the Notes at the end of Chap. 4 in [DDMS].) Hence (HYP) holds in this context. 
This remark in particular shows that we could have worked from the beginning with such simpler $p$-valuations like $\omega^{\prime}$. But in order to have as much flexibility as possible for later applications it seemed advantageous to us to instead minimize the requirements on the $p$-valuation.

Lemma 4.4: Assuming (HYP) we have $\left\|b_{i} b_{j}-b_{j} b_{i}\right\|_{r}<\left\|b_{i} b_{j}\right\|_{r}$ for any $1 \leq$ $i<j \leq d$ and any $1 / p<r<1$.

Proof: Put $c:=\omega\left(h_{i}\right)+\omega\left(h_{j}\right)$. Since $i<j$ we have $\left\|b_{i} b_{j}\right\|_{r}=r^{c}$. To estimate the left hand side in the assertion we use that our hypothesis implies that $h:=$ $h_{i}^{-1} h_{j}^{-1} h_{i} h_{j}=g^{p}$ for some $g \in G$. Hence

$$
\begin{aligned}
b_{i} b_{j}-b_{j} b_{i} & =h_{i} h_{j}-h_{j} h_{i}=h_{j} h_{i}(h-1) \\
& =h_{j} h_{i}\left(g^{p}-1\right)=h_{j} h_{i}\left(((g-1)+1)^{p}-1\right) \\
& =h_{j} h_{i}(g-1)^{p}+\sum_{n=1}^{p-1}\left(\begin{array}{c}
p \\
n
\end{array}\right) h_{j} h_{i}(g-1)^{n}
\end{aligned}
$$

and therefore, by the submultiplicativity of \|\|$_{r}$ and the fact that $\left\|h_{i}\right\|_{r}=1$,

$$
\left\|b_{i} b_{j}-b_{j} b_{i}\right\|_{r} \leq \max \left(\|g-1\|_{r}^{p},|p|\|g-1\|_{r}\right) \text {. }
$$

Since $1 / p \leq r<1$ the inequality $\|g-1\|_{1 / p} \leq p^{-\omega(g)}$ implies that $\|g-1\|_{r} \leq$ $r^{\omega(g)}$. To see this let $0<z \leq 1$ such that $(1 / p)^{z}=r$ and consider the expansion $g-1=\sum_{\alpha} d_{\alpha} \mathbf{b}^{\alpha}$. We have $\left|d_{\alpha}\right| p^{-\tau \alpha} \leq p^{-\omega(g)}$. By exponentiating this latter inequality and using that $\left|d_{\alpha}\right| \leq 1$ we have

$$
\left|d_{\alpha}\right| r^{\tau \alpha} \leq\left|d_{\alpha}\right|^{z} p^{-z \tau \alpha} \leq p^{-z \omega(g)}=r^{\omega(g)}
$$

Combining this with the previous estimate we obtain

$$
\left\|b_{i} b_{j}-b_{j} b_{i}\right\|_{r} \leq \max \left(r^{p \omega(g)}, p^{-1} r^{\omega(g)}\right) .
$$

¿From the properties of a $p$-valuation we deduce that

$$
\omega(g)=\omega(h)-1 \geq \omega\left(h_{i}\right)+\omega\left(h_{j}\right)-1=c-1 .
$$

We therefore get

$$
\left\|b_{i} b_{j}-b_{j} b_{i}\right\|_{r} \leq \max \left(r^{p(c-1)}, p^{-1} r^{c-1}\right) .
$$

The inequality $p^{-1} r^{c-1}<r^{c}$ is obvious from $1 / p<r$. It remains to be seen that $c<p(c-1)$. But this is equivalent to $p /(p-1)<c$ which is part of our assumptions. 
Theorem 4.5: Assuming (HYP) let $1 / p<r<1$ and let $R$ be one of the rings $\mathbb{Z}_{p}[[G]]$ or $D_{r}(G, K)$; we then have:

i. $g r_{r} R$ is a polynomial ring over $\mathbb{F}_{p}[\epsilon]$, resp. gr $K$, in the variables $\sigma_{r}\left(b_{1}\right), \ldots$, $\sigma_{r}\left(b_{d}\right)$; the norm \|\|$_{r}$ on $R$ is multiplicative;

ii. if $r \in p^{\mathbb{Q}}$ then $R$ is a (left and right) noetherian integral domain.

Proof: i. By Lemma 4.3 we only need to consider the case $R=\mathbb{Z}_{p}[[G]]$. We have remarked already that $g r_{r} \mathbb{Z}_{p}[[G]]$ as a module is free over $\mathbb{F}_{p}[\epsilon]$ with basis $\left\{\sigma_{r}\left(\mathbf{b}^{\alpha}\right)\right\}_{\alpha}$. Since $\left\|\mathbf{b}^{\alpha}\right\|_{r}=\prod_{i}\left\|b_{i}\right\|_{r}^{\alpha_{i}}$ we have $\sigma_{r}\left(\mathbf{b}^{\alpha}\right)=\prod_{i} \sigma_{r}\left(b_{i}\right)^{\alpha_{i}}$. Moreover, according to Lemma 4.4 , the $\sigma_{r}\left(b_{i}\right)$ commute with each other. The norm \|\|$_{r}$ has to be multiplicative since $g r_{r} R$ is an integral domain.

ii. Because of our assumptions that $K$ is discretely valued, $\omega$ has rational values, and $r \in p^{\mathbb{Q}}$ the filtration $F_{r} R$ is quasi-integral. The assertion therefore is an immediate consequence of i. and Prop. 1.1.

Recall that $L$ denotes an arbitrary finite extension of $\mathbb{Q}_{p}$ contained in $K$.

Remark 4.6: $\mathbb{Z}_{p}[[G]], L[[G]]$, and $D_{1 / p}(G, K)$ are (left and right) noetherian integral domains with multiplicative norm \|\|$_{1 / p}$.

Proof: Since $L$ is a finitely generated $\mathbb{Z}_{p}$-algebra the case $L[[G]]$ follows from the case $\mathbb{Z}_{p}[[G]]$. For the other two cases, using again Prop. 1.1 and Lemma 4.3 , it suffices to know that $g r_{1 / p} \mathbb{Z}_{p}[[G]]$ is a (left and right) noetherian integral domain. But this is already contained in [Laz] since it is shown there, as we have recalled above, that $g r_{1 / p} \mathbb{Z}_{p}[[G]] \cong U(\operatorname{gr}(G))$.

Turning to flatness properties we will call in the following a unital ring homomorphism $R \longrightarrow R^{\prime}$ (faithfully) flat if $R^{\prime}$ is (faithfully) flat as a left as well as a right $R$-module.

Proposition 4.7: Assuming (HYP) the maps

$$
\mathbb{Z}_{p}[[G]] \longrightarrow L[[G]] \longrightarrow D_{r}(G, K)
$$

are flat for any $1 / p \leq r<1$ such that $r \in p^{\mathbb{Q}}$.

Proof: The first map is flat as a base extension of the flat map $\mathbb{Z}_{p} \hookrightarrow L$. To see that the composite map is flat it suffices by Prop. 1.2 and Thm. 4.5.i (resp. the argument in the proof of Remark 4.6 in case $r=1 / p$ ) to check that the associated graded map for the filtration $F_{r}^{*}$ is flat. This latter map, by Lemma 4.3, is a base extension of the map $\mathbb{F}_{p}[\epsilon] \longrightarrow g r \cdot K=k\left[\epsilon_{\mathrm{o}}, \epsilon_{\mathrm{o}}^{-1}\right]$ which obviously is flat. To finally deduce the flatness of the second map it remains to observe that due to the existence of the trace map $L \longrightarrow \mathbb{Q}_{p}$ the tensor product 
$D_{r}(G, K) \otimes_{L[[G]]} M$, for any $L[[G]]$-module $M$, is naturally a direct summand of $D_{r}(G, K) \otimes_{\mathbb{Q}_{p}[[G]]} M=D_{r}(G, K) \otimes_{\mathbf{z}_{p}[[G]]} M$.

Lemma 4.8: Assume (HYP) and let $1 / p<r<1$ be in $p^{\mathbb{Q}}$; then $D_{<r}(G, K)$ is (left and right) noetherian and the map $D_{r}(G, K) \longrightarrow D_{<r}(G, K)$ is flat.

Proof: Both assertions can be checked after a faithfully flat base extension. In particular we may replace the field $K$ by any of its finite extensions. This allows us to assume that the absolute ramification index $e$ of $K$ has the property that

$$
r^{\omega\left(h_{i}\right)}=p^{-m_{i} / e} \text { for } 1 \leq i \leq d \text { and appropriate } m_{i} \in \mathbb{N}
$$

As before let $k$ denote the residue class field of $K$ and $\epsilon_{\mathrm{o}}$ the principal symbol of a prime element $\pi$ of $K$. By Prop. 1.1 and 1.2 it suffices to show that the induced map between the graded rings $g r_{r} D_{r}(G, K) \longrightarrow g r_{r}^{\cdot} D_{<r}(G, K)$ is a flat map between noetherian rings. The left hand graded ring has been computed in Thm. 4.5.i. Our above assumption on $K$ means that the values of the norm \|\|$_{r}$ on $D_{<r}(G, K)$ lie in $|\pi|^{\mathbf{Z}} \cup\{0\}$. Hence

$$
g r_{r}^{\cdot} D_{<r}(G, K)=g r^{\cdot} K \otimes_{k} g r_{r}^{0} D_{<r}(G, K)
$$

Since $g r \cdot K$ is a finitely generated $k$-algebra we are reduced to showing that the map $g r_{r}^{0} D_{r}(G, K) \longrightarrow g r_{r}^{0} D_{<r}(G, K)$ is a flat map between noetherian rings. By definition $F_{r}^{0} D_{<r}(G, K)$ consists of all series of $\sum_{\alpha} d_{\alpha} \mathbf{b}^{\alpha}$ where $\left|d_{\alpha}\right| r^{\tau \alpha} \leq 1$. But $r^{\tau \alpha}=|\pi|^{m_{1} \alpha_{1}+\ldots+m_{d} \alpha_{d}}$. Hence, equivalently, $F_{r}^{0} D_{<r}(G, K)$ is all series $\sum_{\alpha} e_{\alpha}\left(b_{1} / \pi^{m_{1}}\right)^{\alpha_{1}} \ldots\left(b_{d} / \pi^{m_{d}}\right)^{\alpha_{d}}$ where $\left|e_{\alpha}\right| \leq 1$. Mapping this series to $\sum_{\alpha}\left(e_{\alpha} \bmod \pi\right) u_{1}^{\alpha_{1}} \ldots u_{d}^{\alpha_{d}}$ induces a bijection

$$
g r_{r}^{0} D_{<r}(G, K) \stackrel{\cong}{\longrightarrow} k\left[\left[u_{1}, \ldots, u_{d}\right]\right]
$$

into the formal power series over $k$ in the variables $u_{1} \ldots, u_{d}$. By Lemma 4.4 this is an isomorphism of rings. Clearly $g r_{r}^{0} D_{r}(G, K)$ corresponds to the polynomial ring $k\left[u_{1}, \ldots, u_{d}\right]$. It is well known that the inclusion of the polynomial ring into the formal power series ring over a field is a flat map between noetherian rings.

Theorem 4.9: Assuming (HYP) the map $D_{r}(G, K) \longrightarrow D_{r^{\prime}}(G, K)$ is flat for any $1 / p<r^{\prime} \leq r<1$ in $p^{\mathbb{Q}}$.

Proof: We certainly may assume that $r^{\prime}<r$. Then the map in question can be viewed as the composite

$$
D_{r}(G, K) \longrightarrow D_{<r}(G, K) \longrightarrow D_{r^{\prime}}(G, K) .
$$


Because of Lemma 4.8 it remains to be seen that the second map is flat. Again we may enlarge the field $K$ if convenient. This time we assume that the absolute ramification index $e$ of $K$ satisfies

$$
r^{\omega\left(h_{i}\right)}=p^{-m_{i} / e} \quad \text { and } \quad\left(r^{\prime}\right)^{\omega\left(h_{i}\right)}=p^{-m_{i}^{\prime} / e} \quad \text { for } 1 \leq i \leq d
$$

and appropriate $m_{i}<m_{i}^{\prime}$ in $\mathbb{N}$. Since $D_{<r}(G, K)=\mathbb{Q}_{p} \otimes_{\mathbf{Z}_{p}} F_{r}^{0} D_{<r}(G, K)$ it suffices to show that the inclusion $F_{r}^{0} D_{<r}(G, K) \longrightarrow D_{r^{\prime}}(G, K)$ is flat. The advantage of this is that the left term is closed (in fact c-compact) in the Banach space on the right. This means that on both sides the filtration $F_{r^{\prime}}$ is complete. By Prop. 1.2 we therefore are reduced to showing that the corresponding injective graded map $g r_{r^{\prime}} F_{r}^{0} D_{<r}(G, K) \longrightarrow g r_{r^{\prime}} D_{r^{\prime}}(G, K)$ is a flat map between noetherian rings. By the assumption on $K$ on both sides a homogeneous component can be nonzero only if its degree lies in $\frac{1}{e} \mathbb{Z}$. Fix a prime element $\pi$ of $K$, and let $m \in \mathbb{Z}$. Then $F_{r^{\prime}}^{m / e} F_{r}^{0} D_{<r}(G, K)$ consists of all series $\sum_{\alpha} e_{\alpha}\left(b_{1} / \pi^{m_{1}^{\prime}}\right)^{\alpha_{1}} \ldots\left(b_{d} / \pi^{m_{d}^{\prime}}\right)^{\alpha_{d}}$ where $\left|e_{\alpha}\right| \leq \min \left(|\pi|^{m},|\pi|^{\sum_{i}\left(m_{i}^{\prime}-m_{i}\right) \alpha_{i}}\right)$. On the other hand $F_{r^{\prime}}^{m / e} D_{r^{\prime}}(G, K)$ consists of all such series for which $\left|e_{\alpha}\right| \leq|\pi|^{m}$ and $\left|e_{\alpha}\right| \longrightarrow 0$ as $|\alpha| \longrightarrow \infty$. Denoting, as usual, by $k$ the residue class field of $K$ and by $\epsilon_{\mathrm{o}}$ the principal symbol of $\pi$ it follows that

$$
g r_{r^{\prime}}^{m / e} D_{r^{\prime}}(G, K)=\epsilon_{\mathrm{o}}^{m} \cdot k\left[u_{1}, \ldots, u_{d}\right] \quad \text { with } u_{i}:=\epsilon_{\mathrm{o}}^{-m_{i}^{\prime}} \cdot \sigma_{r^{\prime}}\left(b_{i}\right)
$$

and

$$
\begin{aligned}
& g r_{r^{\prime}}^{m / e} F_{r}^{0} D_{<r}(G, K)=\bigoplus\left\{\epsilon_{\mathrm{o}}^{m} \cdot k u_{1}^{\alpha_{1}} \ldots u_{d}^{\alpha_{d}}: \sum_{i}\left(m_{i}^{\prime}-m_{i}\right) \alpha_{i} \leq m\right\}= \\
& \bigoplus\left\{k \epsilon_{\mathrm{o}}^{m-\sum_{i}\left(m_{i}^{\prime}-m_{i}\right) \alpha_{i}}\left(\epsilon_{\mathrm{o}}^{m_{1}^{\prime}-m_{1}} u_{1}\right)^{\alpha_{1}} \ldots\left(\epsilon_{\mathrm{o}}^{m_{d}^{\prime}-m_{d}} u_{d}\right)^{\alpha_{d}}: \sum_{i}\left(m_{i}^{\prime}-m_{i}\right) \alpha_{i} \leq m\right\} .
\end{aligned}
$$

Note that always $\sum_{i}\left(m_{i}^{\prime}-m_{i}\right) \alpha_{i} \geq 0$. We obtain

$$
g r_{r^{\prime}}^{\cdot} D_{r^{\prime}}(G, K)=k\left[\epsilon_{\mathrm{o}}, \epsilon_{\mathrm{o}}^{-1}, u_{1}, \ldots, u_{d}\right]
$$

(compare Thm. 4.5.i) and

$$
g r_{r^{\prime}} F_{r}^{0} D_{<r}(G, K)=k\left[\epsilon_{\mathrm{o}}, \epsilon_{\mathrm{o}}^{m_{1}^{\prime}-m_{1}} u_{1}, \ldots, \epsilon_{\mathrm{o}}^{m_{d}^{\prime}-m_{d}} u_{d}\right]
$$

Obviously both rings are noetherian and the inclusion between them, being localization in $\epsilon_{\mathrm{o}}$, is flat.

Theorem 4.10: For any compact locally $\mathbb{Q}_{p}$-analytic group $G$ which carries a p-valuation satisfying $(H Y P)$ the algebra $D(G, K)$ is a Fréchet-Stein algebra.

Proof: This is a consequence of Thm. 4.5.ii and Thm. 4.9. 
To construct our sequence of norms which exhibits $D(G, K)$ as a Fréchet-Stein algebra we made the two choices of a $p$-valuation $\omega$ on $G$ and of a corresponding ordered basis $\left(h_{1}, \ldots, h_{d}\right)$ of $G$. Consider another ordered basis $\left(h_{1}^{\prime}, \ldots, h_{d}^{\prime}\right)$ with respect to the same $\omega$. Put $b_{i}^{\prime}:=h_{i}^{\prime}-1, \mathbf{b}^{\prime \alpha}:=b_{1}^{\prime \alpha_{1}} b_{2}^{\prime \alpha_{2}} \cdots b_{d}^{\prime \alpha_{d}}$, and $\tau^{\prime} \alpha:=\sum_{i} \alpha_{i} \omega\left(h_{i}^{\prime}\right)$ for $\alpha=\left(\alpha_{1}, \ldots, \alpha_{d}\right) \in \mathbb{N}_{0}^{d}$. Consider the expansions

$$
\mathbf{b}^{\prime \beta}=\sum_{\alpha} c_{\beta, \alpha} \mathbf{b}^{\alpha}
$$

in $\mathbb{Z}_{p}[[G]]$. Since the norm \|\|$_{1 / p}$ on $\mathbb{Z}_{p}[[G]]$ does not depend on the choice of the ordered basis we have

$$
\omega_{p}\left(c_{\beta, \alpha}\right) \geq \max \left(0, \tau^{\prime} \beta-\tau \alpha\right) .
$$

Similarly as in the proof of Lemma 4.1.ii this implies that

$$
\left|c_{\beta, \alpha}\right| r^{\tau \alpha} \leq r^{\tau^{\prime} \beta}
$$

for any $1 / p \leq r \leq 1$. Expanding an element $\lambda \in D(G, K)$ with respect to both bases as

$$
\lambda=\sum_{\alpha} d_{\alpha} \mathbf{b}^{\alpha}=\sum_{\beta} d_{\beta}^{\prime} \mathbf{b}^{\prime \beta}
$$

we have $d_{\alpha}=\sum_{\beta} d_{\beta}^{\prime} c_{\beta, \alpha}$. It follows that

$$
\sup _{\alpha}\left|d_{\alpha}\right| r^{\tau \alpha} \leq \sup _{\beta}\left|d_{\beta}^{\prime}\right| r^{\tau^{\prime} \beta}
$$

For reasons of symmetry we must in fact have equality. This shows that the norms \|\|$_{r}$ on $D(G, K)$, for $1 / p \leq r<1$, are independent of the choice of the ordered basis.

We complete the list of flatness properties with the following result which answers positively a question raised at the end of $\S 3$ in [ST3].

Theorem 4.11: Assuming (HYP) the map $L[[G]] \longrightarrow D(G, K)$ is faithfully flat.

Proof: The other case being completely analogous we give in the following only the argument for left faithful flatness. We also agree that in this proof all occuring numbers $r$ satisfy, without further mentioning, the conditions $1 / p<$ $r<1$ and $r \in p^{\mathbb{Q}}$.

For the flatness we let $J \subseteq L[[G]]$ be a left ideal. We have to show that the canonical map

$$
D(G, K) \otimes_{L[[G]]} J \longrightarrow D(G, K)
$$


is injective. The ring $L[[G]]$ being noetherian by Remark 4.6 we have that the left hand $D(G, K)$-module is finitely presented and hence coadmissible. It follows that

$$
\begin{aligned}
D(G, K) \otimes_{L[[G]]} J & ={\underset{\leftarrow}{r}}_{\lim _{r}} D_{r}(G, K) \otimes_{D(G, K)}\left(D(G, K) \otimes_{L[[G]]} J\right) \\
& =\overleftarrow{\leftarrow}_{r} D_{r}(G, K) \otimes_{L[[G]]} J .
\end{aligned}
$$

But $D_{r}(G, K)$ is flat over $L[[G]]$ according to Thm. 4.7 so that the map $D_{r}(G, K) \otimes_{L[[G]]} J \longrightarrow D_{r}(G, K)$ is injective. Since the projective limit is left exact this implies the injectivity we want.

For faithful flatness we have to show that $D(G, K) \otimes_{L[[G]]} M \neq 0$ for any nonzero $L[[G]]$-module $M$. Let $o$ denote the ring of integers in $L$ and put $o[[G]]:=$ $o \otimes_{\mathbf{z}_{p}} \mathbb{Z}_{p}[[G]]$. Since $L[[G]] \otimes_{o[[G]]} M=L \otimes_{o} M=M$ and hence $D(G, K) \otimes_{L[[G]]}$ $M=D(G, K) \otimes_{o[[G]]} M$ it suffices to show that $D(G, K) \otimes_{o[[G]]} M \neq 0$ for any nonzero $p$-torsionfree $o[[G]]$-module $M$. By the usual argument this reduces to the claim that $D(G, K) \cdot J \neq D(G, K)$ for any proper left ideal $J \subseteq o[[G]]$ such that $o[[G]] / J$ is $p$-torsionfree. Since $o[[G]]$ is noetherian by Remark 4.6 and $o[[G]] \longrightarrow D(G, K)$ is already known to be flat the $D(G, K)$-module $D(G, K)$. $J=D(G, K) \otimes_{o[[G]]} J$ is coadmissible which implies that

$$
D(G, K) \cdot J=\lim _{r} D_{r}(G, K) \otimes_{o[[G]]} J={\underset{\lim }{r}}_{r} D_{r}(G, K) \cdot J
$$

the second identity coming from the fact that $D_{r}(G, K)$ is flat over $o[[G]]$ by Thm. 4.7. It therefore suffices to find at least one $r$ such that $D_{r}(G, K) \cdot J \neq$ $D_{r}(G, K)$.

To simplify the notation we use in the following the abbreviations $R:=o[[G]]$ and $D_{r}:=D_{r}(G, K)$. Giving the outer terms of the exact sequence

$$
0 \longrightarrow D_{r} J \longrightarrow D_{r} \longrightarrow D_{r} / D_{r} J \longrightarrow 0 \text {. }
$$

the filtration induced by $F_{r} D_{r}$ we obtain the exact sequence of associated graded $g r_{r} D_{r}$-modules

$$
0 \longrightarrow g r_{r}^{\circ} D_{r} J \longrightarrow g r_{r}^{\circ} D_{r} \longrightarrow g r_{r}^{\circ}\left(D_{r} / D_{r} J\right) \longrightarrow 0 .
$$

We compare this with the exact sequence of $g r_{r} R$-modules

$$
0 \longrightarrow g r_{r}^{\cdot} J \longrightarrow g r_{r}^{\cdot} R \longrightarrow g r_{r}^{\cdot}(R / J) \longrightarrow 0
$$

arising from giving the outer terms of the exact sequence

$$
0 \longrightarrow J \longrightarrow R \longrightarrow R / J \longrightarrow 0
$$


the filtration induced by $F_{r}^{\cdot} R$. By Lemma 4.3 the map $g r_{r}^{\cdot} R \longrightarrow g r_{r}^{\cdot} D_{r}$ is flat. Hence we have the commutative exact diagram:

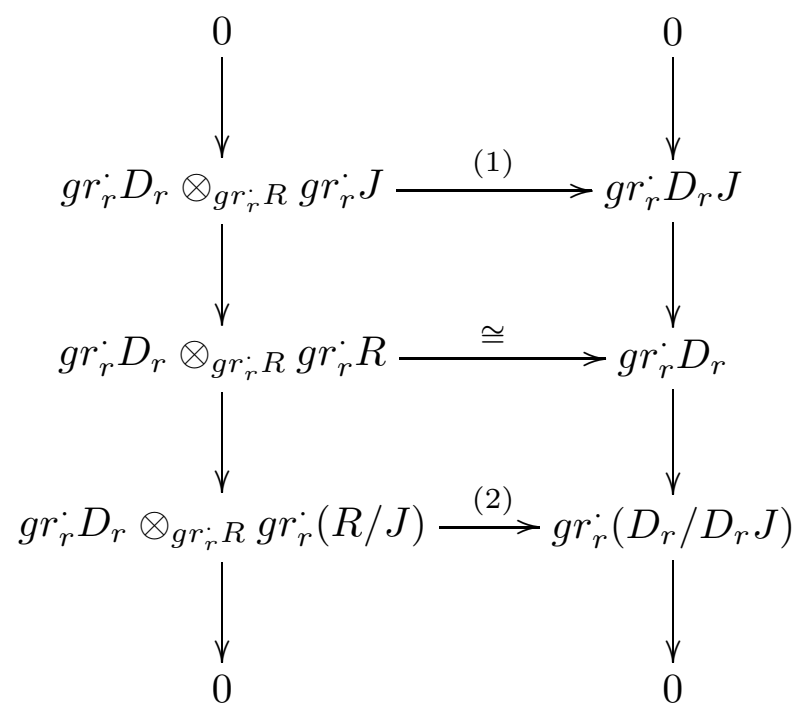

The horizontal arrow in the middle obviously is an isomorphism. Since both rings, $g r_{r} R$ and $g r_{r} D_{r}$, are noetherian by Thm. 4.5.i we may apply Lemma 1.3 and obtain that (1) and hence (2) is an isomorphism. This reduces us to finding an $r$ such that $g r_{r}^{\cdot} D_{r} \otimes_{g r_{r} R} g r_{r}^{\cdot}(R / J) \neq 0$. Since $R$ is compact the finitely generated left ideal $J$ is closed. Hence the filtration $F_{r}(R / J)$ is separated and $g r_{r}(R / J) \neq 0$ if $R / J \neq 0$. From Lemma 4.3 we know that $g r_{r} D_{r}$ is faithfully flat over $\left(g r_{r} R\right)\left[\epsilon^{-1}\right]$. So we are finally reduced to proving that

$$
g r_{r}(R / J) \text { is } \epsilon \text {-torsionfree for } r \text { sufficiently close to } 1
$$

provided $R / J$ is $p$-torsionfree.

Let $\pi \in o$ be a prime element of $L$ and let $e$ denote the absolute ramification index of $L$. It is more convenient to study the multiplication by $\sigma_{r}(\pi)$ (instead of $\left.\epsilon=\sigma_{r}(p)\right)$ on $g r_{r}^{\cdot}(R / J)$. Suppose therefore that

$$
x \in F_{r}^{s} R+J \text { and } \pi x \in F_{r}^{(s+1 / e)+} R+J .
$$

We have to show that $x \in F_{r}^{s+} R+J$ (provided $r$ is sufficiently close to 1 ). Write

$$
\pi x=y+a \quad \text { with } y \in F_{r}^{(s+1 / e)+} R \text { and } a \in J
$$

We will find $y^{\prime} \in R$ and $a^{\prime} \in J \cap F_{r}^{(s+1 / e)+} R$ such that $y=\pi y^{\prime}+a^{\prime}$. Then $\pi y^{\prime}=y-a^{\prime} \in F_{r}^{(s+1 / e)+} R$, hence $y^{\prime} \in F_{r}^{s+} R$, and $\pi\left(x-y^{\prime}\right)=a+a^{\prime} \in J$. Since $R / J$ is assumed to be $\pi$-torsionfree it follows that

$$
x \in y^{\prime}+J \subseteq F_{r}^{s+} R+J .
$$


To actually find $y^{\prime}$ and $a^{\prime}$ we look at the left ideal $J+\pi R / \pi R$ in the ring $R / \pi R$. On $R / \pi R$ we consider the quotient filtration $F_{r}^{s}(R / \pi R):=F_{r}^{s} R+\pi R / \pi R$ and then on $J+\pi R / \pi R$ the induced filtration

$$
F_{r}^{s}(J+\pi R / \pi R):=\left[(J+\pi R) \cap\left(F_{r}^{s} R+\pi R\right)\right] / \pi R .
$$

Since $R$ is compact the quotient filtration on $R / \pi R$ is complete, and $g r_{r}^{\cdot}(R / \pi R)$ being a quotient of $g r_{r}^{\cdot} R$ is noetherian. Hence we may apply Lemma 1.4 to the ideal $J+\pi R / \pi R$ in $R / \pi R$. We obtain elements $a_{1}, \ldots, a_{l} \in J \backslash \pi R$ such that $a_{i}+\pi R \in F_{r}^{s_{i}}(R / \pi R) \backslash F_{r}^{s_{i}+}(R / \pi R)$ and

$$
F_{r}^{s}(J+\pi R / \pi R)=\sum_{i=1}^{l} F_{r}^{s-s_{i}}(R / \pi R) \cdot\left(a_{i}+\pi R\right)
$$

for any $s \in \mathbb{R}$. Since

$$
y+\pi R=-a+\pi R \in F_{r}^{(s+1 / e)+}(J+\pi R / \pi R)
$$

we find $b_{i} \in F_{r}^{\left(s+1 / e-s_{i}\right)+} R$ such that

$$
y+\pi R=b_{1} a_{1}+\ldots+b_{l} a_{l}+\pi R
$$

or equivalently

$$
y=\pi y^{\prime}+a^{\prime} \quad \text { for } a^{\prime}:=b_{1} a_{1}+\ldots+b_{l} a_{l} \in J \text { and some } y^{\prime} \in R .
$$

The element $a^{\prime}$ will lie in $F_{r}^{(s+1 / e)+} R$ provided we have $a_{i} \in F_{r}^{s_{i}} R$ for any $1 \leq i \leq l$. But the subsequent lemma says that this automatically holds true for any $r$ sufficiently close to 1 .

Lemma 4.12: Let $o$ denote the ring of integers in $L$ and $\pi \in o$ a prime element and put $R:=o \otimes \mathbf{z}_{p} \mathbb{Z}_{p}[[G]]$. For any $a \in R \backslash \pi R$ there is a $1 / p \leq r(a)<1$ such that for all $r(a) \leq r<1$ and all $s \in \mathbb{R}$ we have: If $a \in F_{r}^{s} R+\pi R$ then $a \in F_{r}^{s} R$.

Proof: Write $a=\sum_{\alpha} d_{\alpha} \mathbf{b}^{\alpha}$ with $d_{\alpha} \in o$. By assumption we have $\left|d_{\alpha}\right|=1$ for some $\alpha$. We choose a $\beta$ such that $\tau \beta$ is minimal in the set $\left\{\tau \alpha:\left|d_{\alpha}\right|=1\right\}$. For any $\alpha$ with $\tau \alpha \geq \tau \beta$ we have $\left|d_{\alpha}\right| r^{\tau \alpha} \leq r^{\tau \beta}$ for any $r<1$. Consider on the other hand the finitely many $\alpha_{1}, \ldots, \alpha_{l}$ such that $\tau \alpha_{i}<\tau \beta$. Since $\left|d_{\alpha_{i}}\right|<1$ we find a $1 / p \leq r(a)<1$ such that $\left|d_{\alpha_{i}}\right| \leq r(a)^{\tau \beta-\tau \alpha_{i}}$ for any $1 \leq i \leq l$. It follows that $\left|d_{\alpha}\right| r^{\tau \alpha} \leq r^{\tau \beta}$ for any $\alpha$ and any $r(a) \leq r<1$ and hence that

$$
\|a\|_{r} \leq r^{\tau \beta} \quad \text { for any } r(a) \leq r<1 .
$$


Suppose now that $a \in F_{r}^{s} R+\pi R$ for some $r(a) \leq r<1$. Then $\left|d_{\alpha}\right| r^{\tau \alpha} \leq p^{-s}$ for any $\alpha$ such that $\left|d_{\alpha}\right|=1$. In particular $r^{\tau \beta}=\left|d_{\beta}\right| r^{\tau \beta} \leq p^{-s}$ and hence $\|a\|_{r} \leq p^{-s}$.

\section{Distribution algebras of compact Lie groups}

As before let $\mathbb{Q}_{p} \subseteq L \subseteq K \subseteq \mathbb{C}_{p}$ be complete intermediate fields where $L / \mathbb{Q}_{p}$ is finite and $K$ is discretely valued, and let $G$ be an arbitrary compact locally $L$-analytic group. We denote by $G_{0}$ the same group but viewed as a locally $\mathbb{Q}_{p}$-analytic group.

Theorem 5.1: $D(G, K)$ is a Fréchet-Stein algebra.

Proof: Step 1: We first show that $D\left(G_{0}, K\right)$ is a Fréchet-Stein algebra. For this we choose an open normal subgroup $H_{0} \subseteq G_{0}$ which is a uniform pro-p-group. According to [DDMS] $\S 4.2$ the lower $p$-series (loc. cit. Def. 1.15 and Cor. 1.20) in $H_{0}$ is the filtration corresponding to an integrally valued $p$-valuation $\omega$ on $H_{0}$ (in fact, for $p=2$ we have to replace $H_{0}$ by its Frattini subgroup which is the first step in its lower $p$-series so that the restriction of $\omega$ satisfies the axioms of a $p$-valuation). Let $\left(h_{1}, \ldots, h_{d}\right)$ be an ordered basis of $H_{0}$ corresponding to this $\omega$. Since the lower $p$-series consists of characteristic subgroups we have

$$
\omega\left(g h g^{-1}\right)=\omega(h) \quad \text { for any } g \in G_{0}, h \in H_{0} \text {. }
$$

Hence $\left(g h_{1} g^{-1}, \ldots, g h g^{-1}\right)$, for any $g \in G_{0}$, is an ordered basis as well. Let \|\|$_{r}$, for $1 / p<r<1$, be the family of submultiplicative norms on $D\left(H_{0}, K\right)$, corresponding to $\omega$ and $\left(h_{1}, \ldots, h_{d}\right)$, which we have constructed in section 4 . As discussed after Thm. 4.10 each of these norms is in fact independent of the choice of the ordered basis. This means that we have

$$
\left\|\delta_{g} \lambda \delta_{g^{-1}}\right\|_{r}=\|\lambda\|_{r} \quad \text { for any } g \in G_{0} \text { and } \lambda \in D\left(H_{0}, K\right) \text {. }
$$

We now fix coset representatives $1=g_{1}, g_{2}, \ldots, g_{l}$ for $H_{0}$ in $G_{0}$. Then the Dirac distributions $\delta_{g_{1}}, \ldots, \delta_{g_{l}}$ form a basis of $D\left(G_{0}, K\right)$ as a left $D\left(H_{0}, K\right)$-module. For any $\mu \in D\left(G_{0}, K\right)$ write $\mu=\lambda_{1}(\mu) \delta_{g_{1}}+\ldots+\lambda_{l}(\mu) \delta_{g_{l}}$ and put

$$
q_{r}(\mu):=\max \left(\left\|\lambda_{1}(\mu)\right\|_{r}, \ldots,\left\|\lambda_{l}(\mu)\right\|_{r}\right)
$$

Since $D\left(G_{0}, K\right)$ is topologically isomorphic to $D\left(H_{0}, K\right)^{l}$ by the open mapping theorem this defines a continuous norm $q_{r}$ on $D\left(G_{0}, K\right)$ which extends the norm \|\|$_{r}$ on $D\left(H_{0}, K\right)$. It also is clear that the family $q_{r}$, for $1 / p<r<1$, defines the Fréchet topology of $D\left(G_{0}, K\right)$. 
We claim that the multiplication in $D\left(G_{0}, K\right)$ is continuous with respect to each norm $q_{r}$. For any two $\mu, \mu^{\prime} \in D\left(G_{0}, K\right)$ we compute

$$
\begin{aligned}
q_{r}\left(\mu \mu^{\prime}\right) & =q_{r}\left(\sum_{i, j} \lambda_{i}(\mu) \delta_{g_{i}} \lambda_{j}\left(\mu^{\prime}\right) \delta_{g_{j}}\right) \\
& =q_{r}\left(\sum_{i, j} \lambda_{i}(\mu)\left(\delta_{g_{i}} \lambda_{j}\left(\mu^{\prime}\right) \delta_{g_{i}^{-1}}\right) \delta_{g_{i} g_{j}}\right) \\
& =q_{r}\left(\sum_{k} \sum_{i, j} \lambda_{i}(\mu)\left(\delta_{g_{i}} \lambda_{j}\left(\mu^{\prime}\right) \delta_{g_{i}^{-1}}\right) \lambda_{k}\left(\delta_{g_{i} g_{j}}\right) \delta_{g_{k}}\right) \\
& \leq \max _{i, j, k}\left\|\lambda_{i}(\mu)\right\|_{r}\left\|\delta_{g_{i}} \lambda_{j}\left(\mu^{\prime}\right) \delta_{g_{i}^{-1}}\right\|_{r}\left\|\lambda_{k}\left(\delta_{g_{i} g_{j}}\right)\right\|_{r} \\
& =\max _{i, j, k}\left\|\lambda_{i}(\mu)\right\|_{r}\left\|\lambda_{j}\left(\mu^{\prime}\right)\right\|_{r}\left\|\lambda_{k}\left(\delta_{g_{i} g_{j}}\right)\right\|_{r} \\
& \leq \max _{i, j, k}\left\|\lambda_{k}\left(\delta_{g_{i} g_{j}}\right)\right\|_{r} \cdot q_{r}(\mu) \cdot q_{r}\left(\mu^{\prime}\right) .
\end{aligned}
$$

It follows that the completion $D_{r}\left(G_{0}, K\right)$ of $D\left(G_{0}, K\right)$ with respect to $q_{r}$ is a $K$-Banach algebra which contains $D_{r}\left(H_{0}, K\right)$ and which as a left $D_{r}\left(H_{0}, K\right)$ module is free with basis $\delta_{g_{1}}, \ldots, \delta_{g_{l}}$. Hence Thm. 4.5.ii implies that $D_{r}\left(G_{0}, K\right)$ is left noetherian provided $r \in p^{\mathbb{Q}}$. Moreover, since

$$
D_{r^{\prime}}\left(G_{0}, K\right)=D_{r^{\prime}}\left(H_{0}, K\right) \underset{D_{r}\left(H_{0}, K\right)}{\otimes} D_{r}\left(G_{0}, K\right)
$$

as bimodules we obtain from Thm. 4.9 that $D_{r^{\prime}}\left(G_{0}, K\right)$ is flat as a right $D_{r}\left(G_{0}, K\right)$-module for any $1 / p<r^{\prime} \leq r<1$ in $p^{\mathbb{Q}}$. (We note that the above argument is left-right symmetric; in particular $D_{r}\left(G_{0}, K\right)$ is left and right noetherian if $r \in p^{\mathbb{Q}}$.)

Step 2: To derive our assertion from Step 1 we consider the obvious injective map between vector spaces of locally analytic functions

$$
C^{a n}(G, K) \hookrightarrow C^{a n}\left(G_{0}, K\right)
$$

It is in fact a topological embedding (see the proof of [ST4] Lemma 1.2). By the Hahn-Banach theorem and the open mapping theorem we therefore obtain dually a quotient map of Fréchet algebras

$$
D\left(G_{0}, K\right) \longrightarrow D(G, K) \text {. }
$$

Hence the assertion follows from the first step and Prop. 3.7.

Theorem 5.2: The map $L[[G]] \longrightarrow D\left(G_{0}, K\right)$ is faithfully flat.

Proof: Choose an open normal subgroup $H_{0} \subseteq G_{0}$ which is a uniform pro- $p$ group. We then have the bimodule isomorphism

$$
D\left(G_{0}, K\right) \cong L[[G]] \underset{L\left[\left[H_{0}\right]\right]}{\otimes} D\left(H_{0}, K\right)
$$


Hence the assertion follows by base change (compare [B-CA] I§3.3 Prop. 5) from Thm. 4.11.

It is likely that also the map $L[[G]] \longrightarrow D(G, K)$ is faithfully flat. But this seems to require additional arguments beyond the present methods.

\section{Admissible representations}

With $\mathbb{Q}_{p} \subseteq L \subseteq K \subseteq \mathbb{C}_{p}$ as in the previous sections we now let $G$ be an arbitrary locally $L$-analytic group.

Definition: $A$ (left) $D(G, K)$-module is called coadmissible if it is coadmissible as a $D(H, K)$-module for every compact open subgroup $H \subseteq G$.

For any two compact open subgroups $H \subseteq H^{\prime} \subseteq G$ the algebra $D\left(H^{\prime}, G\right)$ is finitely generated free and hence coadmissible as a $D(H, K)$-module. It therefore follows from Lemma 3.8 that the condition in the above definition needs to be tested only for a single compact open subgroup $H$. We let $\mathcal{C}_{G}$ denote the full subcategory of all coadmissible $D(G, K)$-modules in $\operatorname{Mod}(D(G, K))$. As a consequence of Cor. 3.4 it is closed with respect to the formation of finite direct sums, kernels, and cokernels. In particular $\mathcal{C}_{G}$ is an abelian category. A module $M$ in $\mathcal{C}_{G}$ viewed as a $D(H, K)$-module for some compact open subgroup $H \subseteq G$ carries its canonical Fréchet topology. The arguments in the proof of Lemma 3.8 applied to the algebra homomorphism $D(H, K) \longrightarrow D\left(H^{\prime}, K\right)$ show that this topology in fact is independent of the choice of $H$. We therefore call it the canonical topology on $M$. Consider, for any $g \in G$, the map $M \stackrel{g \text {. }}{\longrightarrow} M$. If we view the source as a $D(H, K)$-module and the target as a $D\left(\mathrm{gHg}^{-1}, K\right)$-module then this map is compatible with these module structures relative to the isomorphism of Fréchet algebras $\delta_{g} \cdot \delta_{g^{-1}}: D(H, K) \stackrel{\cong}{\longrightarrow} D\left(g H^{-1}, K\right)$. Using Lemma 3.8 we conclude that this map $M \stackrel{g \cdot}{\longrightarrow} M$ is continuous for the canonical topology. Since

$$
D(G, K)=\bigoplus_{g \in H \backslash G} D(H, K) \delta_{g}
$$

as locally convex vector spaces it then also follows that the $D(G, K)$-action on $M$ is separately continuous. Moreover, any map in $\mathcal{C}_{G}$ is continuous and strict for the canonical topologies.

Lemma 6.1: The canonical topology on a coadmissible $D(G, K)$-module $M$ is nuclear. 
Proof: We choose a compact open subgroup $H \subseteq G$ such that the locally $\mathbb{Q}_{p^{-}}$ analytic group $H_{0}$ which underlies $H$ carries a $p$-valuation $\omega$ satisfying the condition (HYP) in section 4 . We also fix an ordered basis $\left(h_{1}, \ldots, h_{d}\right)$ of $\left(H_{0}, \omega\right)$ and denote by \|\|$_{r}$, for $1 / p \leq r<1$, the family of norms on $D\left(H_{0}, K\right)$ constructed in section 4 . The completion of $D\left(H_{0}, K\right)$, resp. of the quotient algebra $D(H, K)$, with respect to \|\|$_{r}$, resp. the quotient norm of \|\|$_{r}$, is denoted by $D_{r}\left(H_{0}, K\right)$, resp. $D_{r}(H, K)$. As a Fréchet space $M$ is the projective limit

$$
M=\lim _{r} M_{r}
$$

of the Banach spaces

$$
M_{r}:=D_{r}(H, K) \underset{D(H, K)}{\otimes} M .
$$

For its nuclearity it suffices to check (compare [NFA] Prop. 19.9) that the transition maps $M_{r} \longrightarrow M_{r^{\prime}}$, for $1 / p \leq r^{\prime}<r<1$, are compact. But we have, for some $l \in \mathbb{N}$, a commutative diagram

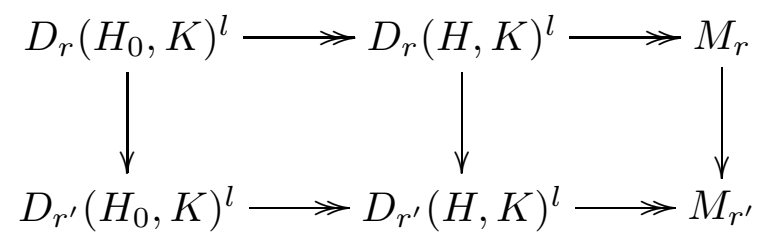

where the horizontal maps are strict surjections. Hence it suffices to show that the first perpendicular arrow is compact. In other words we are reduced to showing that the natural map

$$
D_{r}\left(H_{0}, K\right) \longrightarrow D_{r^{\prime}}\left(H_{0}, K\right)
$$

is compact. The ring structure being irrelevant here we may use the global chart $\psi$ corresponding to the fixed ordered basis to further reduce this to the compactness of the map

$$
D_{r}\left(\mathbb{Z}_{p}^{d}, K\right) \longrightarrow D_{r^{\prime}}\left(\mathbb{Z}_{p}^{d}, K\right) .
$$

As we have discussed after Prop. 4.2, both sides are Banach spaces of convergent power series on certain closed disks strictly contained in each other and the map is the restriction map. By a well known nonarchimedean version of Montel's theorem (compare [NFA] p.99) this map is compact.

Remark 6.2: Suppose that $G$ is compact; then the analytic $D(G, K)$-modules in the sense of [ST1] 11 are precisely the finitely generated coadmissible $D(G, K)$ modules. 
Proof: If $M$ is a finitely generated and coadmissible $D(G, K)$-module then, in view of its canonical Fréchet topology, it follows from [ST1] Prop. 1.1 that $M$ is analytic. Vice versa, if $M$ is analytic then it is shown in the proof of loc. cit. that $M$ is isomorphic to a quotient of a finitely generated free $D(G, K)$-module (with its canonical topology) by a closed submodule. Hence Lemma 3.6 implies that $M$ is coadmissible.

It follows from the above remark (and Cor. 3.4.v) that for compact $G$ any finitely presented $D(G, K)$-module is analytic. This was posed as an open question in [ST1].

We recall from [ST2] $\S 3$ that a locally analytic $G$-representation $V$ (over $K$ ) is a barrelled locally convex Hausdorff $K$-vector space $V$ equipped with a $G$-action by continuous linear endomorphisms such that, for each $v \in V$, the orbit map $\rho_{v}(g):=g v$ is a $V$-valued locally analytic function on $G$. The $G$-action extends to a separately continuous action of the algebra $D(G, K)$ on $V$. It was proved in [ST2] Cor. 3.3 that the functor $V \longmapsto V_{b}^{\prime}$ of passing to the strong dual induces an anti-equivalence of categories:

locally analytic $G$-representations $\quad$ separately continuous $D(G, K)$ -

on $K$-vector spaces of compact type $\rightarrow \quad$ modules on nuclear Fréchet with continuous linear $G$-maps $\rightarrow \quad$ spaces with continuous

$D(G, K)$-module maps

The objects on the right hand side naturally come as right $D(G, K)$-modules. But using the anti-involution $g \longmapsto g^{-1}$ we may and will view them as left $D(G, K)$-modules.

Definition: An admissible $G$-representation over $K$ is a locally analytic $G$ representation on a $K$-vector space of compact type $V$ such that the strong dual $V_{b}^{\prime}$ is a coadmissible $D(G, K)$-module equipped with its canonical topology.

We let $\operatorname{Rep}_{K}^{a}(G)$ denote the category of all admissible $G$-representations over $K$ with continuous $K$-linear $G$-maps.

Theorem 6.3: The functor

$$
\begin{aligned}
\operatorname{Rep}_{K}^{a}(G) & \stackrel{\sim}{\longrightarrow} \mathcal{C}_{G} \\
V & \longmapsto V^{\prime}
\end{aligned}
$$

is an anti-equivalence of categories.

Proof: Consider the anti-equivalence which we have recalled above from [ST2]. According to Lemma 6.1 equipping a coadmissible $D(G, K)$-module with its 
canonical topology makes $\mathcal{C}_{G}$ into a full subcategory of the right hand side which, by definition, is the essential image of $\operatorname{Rep}_{K}^{a}(G)$ under the functor $V \longmapsto V_{b}^{\prime}$.

Proposition 6.4: $i$. $\operatorname{Rep}_{K}^{a}(G)$ is an abelian category; kernel and image of a morphism in $\operatorname{Rep}_{K}^{a}(G)$ are the algebraic kernel and image with the subspace topology;

ii. any map in $\operatorname{Rep}_{K}^{a}(G)$ is strict and has closed image;

iii. the category $\operatorname{Rep}_{K}^{a}(G)$ is closed with respect to the passage to closed $G$ invariant subspaces.

Proof: $\operatorname{Rep}_{K}^{a}(G)$ is abelian as a consequence of Thm. 6.3. Let $f: V \longrightarrow W$ be a map in $\operatorname{Rep}_{K}^{a}(G)$. The dual map $f^{\prime}: W_{b}^{\prime} \longrightarrow V_{b}^{\prime}$, as a map between coadmissible modules with their canonical topology, is a strict map between nuclear and hence ([NFA] Cor. 19.3.ii) reflexive Fréchet spaces. It then follows from the nonarchimedean analog of [B-TVS] IV $\$ 4.2$ Cor. 3 and the fact that over a nonarchimedean field every reflexive space already is Montel ([NFA] Prop. 15.3) that $f=f^{\prime \prime}$ is strict as well and has closed image. The statement about the kernel and image of $f$ is an easy consequence of this. The assertion iii. finally follows from Lemma 3.6 and [ST2] Prop. 1.2(i) (compare the proof of [ST2] Lemma 3.5).

In [ST2] $\S 3$ we have introduced, for compact groups $G$, the notion of a strongly admissible $G$-representation. This is a locally analytic $G$-representation in a vector space of compact type $V$ such that $V^{\prime}$ is finitely generated as a $D(G, K)$ module. But then $V_{b}^{\prime}$ is a quotient of some $D(G, K)^{l}$ by some closed submodule and hence is coadmissible (with its canonical topology) by Lemma 3.6. It therefore follows from Thm. 6.3 that the strongly admissible $G$-representations are precisely the admissible $G$-representations for which $V^{\prime}$ is a finitely generated $D(G, K)$-module (provided $G$ is compact).

The following technical useful observation says that, for compact $G$ again, the structure of a vector space of compact type which underlies an admissible $G$ representation $V$ in fact can be chosen in a $G$-equivariant way.

Proposition 6.5: Suppose that $G$ is compact; any admissible G-representation $V$ is a compact inductive limit of locally analytic $G$-representations on $K$-Banach spaces $V_{n}$ for $n \in \mathbb{N}$.

Proof: Let $\left(r_{n}\right)_{n \in \mathbb{N}}$ be a sequence of real numbers in $\left\{r \in p^{\mathbb{Q}}: 1 / p<r<1\right\}$ which converges to 1 , and let $A_{n}:=D_{r_{n}}(G, K)$ be the corresponding sequence of Banach algebras constructed in the proof of Thm. 5.1 which realizes the structure of $D(G, K)$ as a Fréchet-Stein algebra. The Fréchet space $V_{b}^{\prime}$ is the projective limit of the projective system

$$
\ldots \longrightarrow M_{n} \longrightarrow \ldots \longrightarrow M_{1}
$$


of Banach spaces $M_{n}:=A_{n} \otimes_{D(G, K)} V^{\prime}$. We let $V_{n}:=M_{n}^{\prime}$ denote the dual Banach spaces. It was shown in the proof of Lemma 6.1 that the transition maps in the above projective system are compact (note that as Banach spaces the $M_{n}$ remain unchanged if $G$ is replaced by an open normal subgroup as we do in that proof). Moreover, by Theorem A, the projection maps $V^{\prime} \longrightarrow M_{n}$ have dense image. It follows that the maps in the dual inductive system

$$
V_{1} \longrightarrow \ldots \longrightarrow V_{n} \longrightarrow \ldots
$$

are injective and compact ([NFA] Lemma 16.4) and that ([NFA] Prop. 16.5)

$$
V=\left(V_{b}^{\prime}\right)_{b}^{\prime}=\underset{n}{\lim } V_{n}
$$

is the compact inductive limit of the $V_{n}$. By construction the original projective system is a system of continuous $D(G, K)$-modules. By functoriality the dual inductive system therefore is $G$-equivariant. It remains to show that the $G$-action on each $V_{n}$ is locally analytic. The continuous $A_{n}$-module structure on $M_{n}$ amounts to a continuous homomorphism $A_{n} \longrightarrow \mathcal{L}_{s}\left(M_{n}, M_{n}\right)$ into the space of bounded operators on $M_{n}$ equipped with the topology of pointwise convergence. By [B-TVS] III.31 Prop. 6 this map $A_{n} \longrightarrow \mathcal{L}_{b}\left(M_{n}, M_{n}\right)$ remains continuous when the target is given the operator norm topology . The dual action of $A_{n}$ on $V_{n}$ arises by composing this map with the passage to the adjoint and therefore is continuous as well. Hence the $G$-action on $V_{n}$ is the restriction of a continuous $D(G, K)$-action. Moreover, the orbit map $\rho_{v}$, for any $v \in V_{n}$, is the image of the continuous linear map $\lambda \longmapsto \lambda v$ in $\mathcal{L}\left(D(G, K), V_{n}\right)$ under the integration isomorphism ([ST2] Thm. 2.2)

$$
\mathcal{L}\left(D(G, K), V_{n}\right) \cong C^{a n}(G, V)
$$

and consequently is locally analytic.

Next we want to discuss how the smooth representation theory of $G$ relates to the category $\operatorname{Rep}_{K}^{a}(G)$. We recall that a smooth $G$-representation $V$ (over $K$ ) is a $K$-vector space $V$ with a linear $G$-action such that the stabilizer of each vector in $V$ is open in $G$. A smooth $G$-representation $V$ is called admissible-smooth if, for any compact open subgroup $H \subseteq G$, the vector subspace $V^{H}$ of $H$-invariant vectors in $V$ is finite dimensional. As we discussed in [ST1] $\S 2$, any admissiblesmooth $G$-representation $V$ equipped with the finest locally convex topology is a locally analytic $G$-representation on a vector space of compact type. The derived action of the Lie algebra $\mathfrak{g}$ of $G$ in this case obviously is trivial.

Theorem 6.6: $i$. Any admissible-smooth $G$-representation $V$ equipped with the finest locally convex topology is admissible and has a trivial derived Lie algebra action; 
ii. any admissible $G$-representation $V$ with trivial derived Lie algebra action is admissible-smooth equipped with the finest locally convex topology.

Proof: We fix a compact open subgroup $H \subseteq G$. Let $I(\mathfrak{g}) \subseteq D(H, K)$ denote the closed two sided ideal generated by $\mathfrak{g}$. The quotient algebra $D^{\infty}(H, K):=$ $D(H, K) / I(\mathfrak{g})$ is the algebra of locally constant distributions on $H$; it is the projective limit

$$
D^{\infty}(H, K)=\lim _{N} K[H / N]
$$

of the algebraic group rings $K[H / N]$ where $N$ runs over the open normal subgroups of $H$ (see [ST1] §2). This latter description visibly exhibits $D^{\infty}(H, K)$ as a $K$-Fréchet-Stein algebra.

i. The strong dual of $V$ when we equip $V$ with the finest locally convex topology is the full linear dual $\operatorname{Hom}_{K}(V, K)$ with the topology of pointwise convergence. Since $\mathfrak{g}$ acts trivially on $V$ the $D(H, K)$-action on $\operatorname{Hom}_{K}(V, K)$ factorizes through $D^{\infty}(H, K)$. As $V$ is the locally convex inductive limit of the finite dimensional vector spaces $V^{N}$ its strong dual satisfies

$$
V_{b}^{\prime}=\lim _{N} \operatorname{Hom}_{K}\left(V^{N}, K\right)
$$

In addition we have

$$
K[H / N] \underset{D^{\infty}(H, K)}{\otimes} \operatorname{Hom}_{K}(V, K)=\operatorname{Hom}_{K}\left(V^{N}, K\right) .
$$

This shows that $V_{b}^{\prime}$ is coadmissible with the canonical topology as a $D^{\infty}(H, K)$ and hence as a $D(H, K)$-module.

ii. The $D(H, K)$-action on $V$ and hence on $V_{b}^{\prime}$ factorizes through $D^{\infty}(H, K)$ by assumption. Hence the $G$-action on $V$ is smooth ([ST1] 22 ). Secondly, as a coadmissible $D^{\infty}(H, K)$-module with the canonical topology $V_{b}^{\prime}$ is the projective limit of the finite dimensional vector spaces $K[H / N] \otimes_{D^{\infty}(H, K)} V^{\prime}$. Dualizing again we obtain that $V \cong\left(V_{b}^{\prime}\right)_{b}^{\prime}$ is a locally convex inductive limit of finite dimensional vector spaces and therefore carries the finest locally convex topology. The finite dimensionality of

$$
K[H / N] \underset{D^{\infty}(H, K)}{\otimes} V^{\prime}=K[H / N] \underset{D^{\infty}(H, K)}{\otimes} \operatorname{Hom}_{K}(V, K)=\operatorname{Hom}_{K}\left(V^{N}, K\right)
$$

finally implies that $V^{N}$ is finite dimensional for each $N$. Hence the $G$-action on $V$ is admissible-smooth.

A vector $v \in V$ in a general admissible $G$-representation $V$ is called smooth if the orbit map $\rho_{v}$ is locally constant on $G$. The smooth vectors form a $G$-invariant 
vector subspace $V_{\text {smooth }}$ in $V$. Taylor's formula shows that a vector $v$ is smooth if and only if $v$ is annihilated by the derived $\mathfrak{g}$-action on $V$. Hence $V_{\text {smooth }}$ coincides with the largest vector subspace of $V$ on which the $\mathfrak{g}$-action is trivial:

$$
V_{\text {smooth }}=V^{\mathfrak{g}=0}
$$

In particular, $V_{\text {smooth }}$ is closed in $V$ and is therefore an admissible $G$-representation by Prop. 6.4.iii. Thm. 6.6.ii then implies that $V_{\text {smooth }}$ in fact is the largest admissible-smooth subrepresentation of $V$.

\section{The existence of analytic vectors}

In this section we will explore the faithful flatness result Thm. 5.2. Throughout we let $G$ be a compact locally $\mathbb{Q}_{p}$-analytic group and we let $K$ be a finite extension of $\mathbb{Q}_{p}$ and $o$ its ring of integers. A $K$-Banach space representation $V$ of $G$ is a $K$-Banach space $V$ together with a continuous linear action $G \times V \longrightarrow V$ of $G$ on $V$. The $G$-action extends naturally to an action of the algebra $K[[G]]$ by continuous linear endomorphisms on $V$; the induced action $o[[G]] \times V \longrightarrow V$ of the compact subring $o[[G]]$ is continuous (see $[\mathrm{ST} 3] \S 2$ ). By functoriality $K[[G]]$ then also acts on the continuous dual $V^{\prime}$. In [ST3] $\S 2$ (in particular Lemma 3.4) we have called a $K$-Banach space representation $V$ of $G$ admissible if the dual $V^{\prime}$ is finitely generated as a $K[[G]]$-module. As in loc. cit. we let $\operatorname{Ban}_{G}^{a d m}(K)$ denote the category of all admissible $K$-Banach space representations of $G$ with morphisms being all $G$-equivariant continuous linear maps. According to [ST3] Thm. 3.5 the functor

$$
\operatorname{Ban}_{G}^{a d m}(K) \stackrel{\sim}{\longrightarrow} \mathcal{M}_{K[[G]]}
$$

is an anti-equivalence of categories.

Since the algebra $K[[G]]$ is noetherian we have the functor

$$
D(G, K) \underset{K[[G]]}{\otimes}:: \mathcal{M}_{K[[G]]} \longrightarrow \mathcal{C}_{G}
$$

By composition we obtain a natural functor

$$
\begin{aligned}
\operatorname{Ban}_{G}^{a d m}(K) & \longrightarrow \operatorname{Rep}_{K}^{a}(G) \\
V & \longmapsto\left(D(G, K) \underset{K[[G]]}{\otimes} V^{\prime}\right)_{b}^{\prime}
\end{aligned}
$$

here the strong dual on the right hand side is formed with respect to the canonical topology on the coadmissible $D(G, K)$-module $D(G, K) \otimes_{K[[G]]} V^{\prime}$. The goal of this section is to give a description of this functor directly in terms of the $K$-Banach space representation $V$. 
Let us fix a $K$-Banach space representation $V$ of $G$.

Definition: $A$ vector $v \in V$ is called analytic if the continuous orbit map $\rho_{v}$ is locally analytic.

We let $V_{a n} \subseteq V$ denote the vector subspace of analytic vectors. It is clearly $G$-invariant. Moreover the $G$-equivariant linear map (with $G$ acting by left translations on the right hand side)

$$
\begin{aligned}
V_{a n} & \longrightarrow C^{a n}(G, V) \\
v & \longmapsto\left[g \mapsto g^{-1} v\right]
\end{aligned}
$$

is injective. We always equip $V_{a n}$ with the subspace topology with respect to this embedding. (Note that this topology in general is strictly finer than the topology induced by $V$.) Of course the $G$-action on $V_{a n}$ is continuous.

Theorem 7.1: For any admissible $K$-Banach space representation $V$ we have:

i. $V_{a n}$ is dense in $V$;

ii. $V_{\text {an }}$ is a strongly admissible $G$-representation;

iii. $\left(V_{a n}\right)^{\prime}=D(G, K) \underset{K[[G]]}{\otimes} V^{\prime}$.

Moreover, the functor

$$
\begin{aligned}
\operatorname{Ban}_{G}^{a d m}(K) & \longrightarrow \operatorname{Rep}_{K}^{a}(G) \\
V & \longmapsto V_{a n}
\end{aligned}
$$

is exact.

Proof: ii. Let $C(G, K)$ denote the $K$-Banach space of continuous functions on $G$. Given finitely many $l_{1}, \ldots, l_{m} \in V^{\prime}$ the $G$-equivariant continuous linear map

$$
\begin{aligned}
& V \longrightarrow C(G, K)^{m} \\
& v \longmapsto\left(\left[g \mapsto l_{i}\left(g^{-1} v\right)\right]\right)_{1 \leq i \leq m}
\end{aligned}
$$

dualizes into the $K[[G]]$-module homomorphism

$$
\begin{aligned}
K[[G]]^{m} & \longrightarrow V^{\prime} \\
\left(\mu_{1}, \ldots, \mu_{m}\right) & \longmapsto \mu_{1} l_{1}+\ldots+\mu_{m} l_{m} .
\end{aligned}
$$

Since, by assumption, $V^{\prime}$ is finitely generated as a $K[[G]]$-module it follows, using the Hahn-Banach theorem, that we may choose the $l_{1}, \ldots, l_{m}$ in such a way that they are $K$-linearly independent and that the map (2) is injective. Its restriction

$$
\begin{aligned}
V_{a n} & \longrightarrow C^{a n}(G, K)^{m} \\
v & \longmapsto
\end{aligned}
$$


then is injective as well. Let us consider the commutative triangle

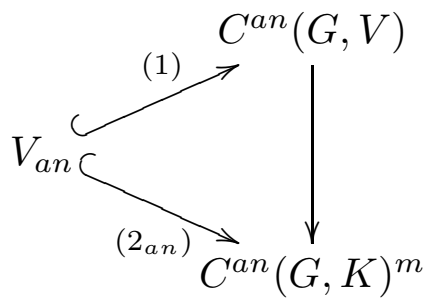

where the perpendicular arrow is induced by the linear forms $l_{1}, \ldots, l_{m}$. By the definition of the topology on $V_{a n}$ and by [ST5] Remark 3.6 the map (1) is a closed embedding. The perpendicular map is continuous and, having a continuous section, closed. It follows that the map $\left(2_{a n}\right)$ is a closed embedding. But $C^{a n}(G, K)^{m}$, according to [Fea] 2.3.2 (alternatively use an argument as in the proof of Lemma 6.1), is a vector space of compact type. Using [Fea] 3.1.7 and [ST2] Prop. 1.2(i) we conclude that $V_{a n}$ is a locally analytic $G$-representation on a vector space of compact type. Moreover, by the Hahn-Banach theorem again, the map $\left(2_{a n}\right)$ dualizes into a $D(G, K)$-linear continuous surjection between Fréchet spaces
$\left(2_{a n}\right)^{\prime}$
$D(G, K)^{m} \longrightarrow\left(V_{a n}\right)_{b}^{\prime}$.

In this situation Lemma 3.6 implies that $\left(V_{a n}\right)_{b}^{\prime}$ is a finitely generated coadmissible $D(G, K)$-module with its canonical topology.

iii. Keeping the above notations we have the commutative diagram:

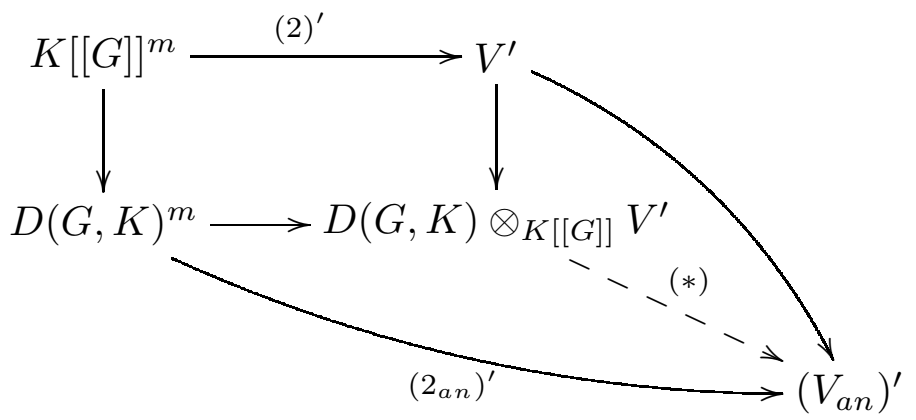

The canonical map $(*)$ which, by ii., lies in $\mathcal{C}_{G}$, is surjective since the map $\left(2_{a n}\right)^{\prime}$ is surjective. It is continuous with respect to the canonical topologies on both sides. Note that the target with the canonical topology coincides with $\left(V_{a n}\right)_{b}^{\prime}$. We define

$$
V_{1}:=\left(D(G, K) \underset{K[[G]]}{\otimes} V^{\prime}\right)_{b}^{\prime}
$$

as an admissible $G$-representation. Using Hahn-Banach we see that $(*)$ is injective if the dual map $V_{a n} \longrightarrow V_{1}$ is surjective. We will establish this by looking 
at the right hand perpendicular arrow. Let $M \subseteq V^{\prime}$ denote the image of $o[[G]]^{m}$ under the map $(2)^{\prime}$. The Fréchet topology of $D(G, K)$ induces on $o[[G]]$ its natural compact topology as a completed group ring. The quotient topology from $o[[G]]^{m}$ on $M$ is the unique (compact) Hausdorff topology on $M$ which makes the module structure $o[[G]] \times M \longrightarrow M$ continuous (compare [ST3] Prop. 3.1). We write $V_{b s}^{\prime}$ for $V^{\prime}$ equipped with the finest locally convex topology such that the inclusion $M \subseteq V^{\prime}$ is continuous. Then the map under consideration is continuous as a map of locally convex vector spaces

$$
V_{b s}^{\prime} \longrightarrow D(G, K) \underset{K[[G]]}{\otimes} V^{\prime}
$$

(where the target, as always, carries its canonical topology). Since $K[[G]]$ is dense in $D(G, K)$ ([ST2] Lemma 3.1) this map has dense image. Hence the dual map $V_{1} \longrightarrow\left(V_{b s}^{\prime}\right)_{b}^{\prime}$ is injective. The discussion before Lemma 1.4 in [ST3] tells us that as vector spaces we have $\left(V_{b s}^{\prime}\right)^{\prime}=V$. We therefore have the two injective maps

$$
V_{a n} \longrightarrow V_{1} \longrightarrow\left(V_{b s}^{\prime}\right)^{\prime}=V
$$

whose composition obviously is the inclusion $V_{a n} \subseteq V$. The left map is continuous by construction. If we show that the right map is continuous with respect to the original Banach space topology on $V$ then its image has to fall into $V_{a n}$ since $V_{1}$ is a locally analytic $G$-representation, and we obtain $V_{a n}=V_{1}$ as claimed. For this continuity it suffices to check that the strong topology on $\left(V_{b s}^{\prime}\right)^{\prime}$ is finer than the Banach space topology on $V$. According to [ST3] the Banach space $V$ is identified with $\operatorname{Hom}_{o}^{\text {cont }}(M, K)$ with norm given by the sup-norm, and the unit ball is $\operatorname{Hom}_{o}^{\text {cont }}(M, o)$. But $M$ is compact and therefore bounded in $V_{b s}^{\prime}$. Hence $\operatorname{Hom}_{o}^{\text {cont }}(M, o)$ is open in $\left(V_{b s}^{\prime}\right)_{b}^{\prime}$.

i. In view of what we know already it suffices to show that the image of this latter continuous map $V_{1} \longrightarrow V$ is dense in $V$. But this is, by Hahn-Banach, a consequence of the injectivity of the dual map $V^{\prime} \longrightarrow D(G, K) \otimes_{K[[G]]} V^{\prime}$ which in turn follows from the faithful flatness of $D(G, K)$ over $K[[G]]$ (Thm. 5.2).

Finally, in order to establish the last assertion, we now have that the functor $V \longmapsto V_{a n}$ of passing to the subspace of analytic vectors coincides with the composite functor:

$$
\begin{aligned}
& \operatorname{Ban}_{G}^{a d m}(K) \quad \longrightarrow \mathcal{M}_{K[[G]]} \quad \longrightarrow \quad \mathcal{C}_{D(G, K)} \quad \longrightarrow \quad \operatorname{Rep}_{K}^{a}(G) \\
& V \quad \longmapsto \quad V^{\prime} \longmapsto D(G, K) \underset{K[[G]]}{\otimes} V^{\prime} \longmapsto\left(D(G, K) \otimes V^{\prime}\right)_{b}^{\prime}
\end{aligned}
$$

In this composition all three functors are exact: The first one by the strictness of all maps in $\operatorname{Ban}_{G}^{a d m}(K)$ ([ST3]) and Hahn-Banach, the second one by Thm. 5.2 , and the third one by the strictness of all maps in $\mathcal{C}_{D(G, K)}$ with respect to the canonical topologies and Hahn-Banach. Hence the functor $V \longmapsto V_{a n}$ on $\operatorname{Ban}_{G}^{a d m}(K)$ is exact. 
The content of this section generalizes in a straightforward way to arbitrary locally $\mathbb{Q}_{p}$-analytic groups $G$ if one extends the notion of an admissible Banach representation from the compact to the general case by a procedure similar to the one we have used in section 6 .

\section{Dimension theory for coadmissible modules}

Over commutative noetherian rings the size of a finitely generated module can be measured by the dimension of its support. If the ring, in addition, is regular then this dimension function can be described in terms of the vanishing of certain Ext-modules. In this latter form dimension theory was generalized to so called Auslander regular noncommutative noetherian rings (compare [Bjo]). The Banach algebras $D_{r}(G, K)$ which we have introduced in section 4 turn out to be Auslander regular. We will demonstrate in the present section that, as a consequence, the category $\mathcal{C}_{G}$, for any locally $\mathbb{Q}_{p}$-analytic group $G$, allows a well behaved dimension theory. We will proceed in an axiomatic way, and we begin by setting up the general formalism of Ext-functors in our context.

We always will assume in this section that $A$ is a "two sided" $K$-Fréchet-Stein algebra by which we mean the existence of a sequence $q_{1} \leq \ldots \leq q_{n} \leq \ldots$ of continuous algebra seminorms on $A$ defining the Fréchet topology of $A$ such that

(i) $A_{q_{n}}$ is left and right noetherian, and

(ii) $A_{q_{n}}$ is flat as a left and as a right $A_{q_{n+1}}$-module for any $n \in \mathbb{N}$.

Definition: $A$ (left module) sheaf for $\left(A,\left(q_{n}\right)\right)$ is a family $S=\left(S_{n}\right)_{n \in \mathbb{N}}$ of left unital $A_{q_{n}}$-modules $S_{n}$ together with $A_{q_{n+1}}$-module homomorphisms $S_{n+1} \longrightarrow$ $S_{n}$ for any $n \in \mathbb{N}$.

These sheaves, together with the obvious notion of a homomorphism, form an abelian category $S h_{\left(A,\left(q_{n}\right)\right)}$. It contains as a full abelian subcategory the category $\operatorname{Coh}_{\left(A,\left(q_{n}\right)\right)}$ of coherent sheaves as introduced in section 3. The left exact global section functor is defined by

$$
\begin{aligned}
\Gamma: S h_{\left(A,\left(q_{n}\right)\right)} & \longrightarrow \operatorname{Mod}(A) \\
S & \longmapsto \lim _{n} S_{n} .
\end{aligned}
$$

According to Cor. 3.3 its restriction to $\operatorname{Coh}_{\left(A,\left(q_{n}\right)\right)}$ is exact and induces an equivalence of categories

$$
\Gamma: \operatorname{Coh}_{\left(A,\left(q_{n}\right)\right)} \stackrel{\sim}{\longrightarrow} \mathcal{C}_{A}
$$


There are obvious right module versions of these notions and facts which we will need later on and for which we will use corresponding notations decorated with a superscript " $r$ ". The following three facts elaborate on the methods in section II of $[\mathrm{Ban}]$.

Lemma 8.1: $i$. The category $S h_{\left(A,\left(q_{n}\right)\right)}$ has enough injective objects;

ii. for any $n_{0} \in \mathbb{N}$ the functor

$$
\begin{aligned}
S h_{\left(A,\left(q_{n}\right)\right)} & \longrightarrow \operatorname{Mod}\left(A_{q_{n_{0}}}\right) \\
\left(S_{n}\right)_{n} & \longmapsto S_{n_{0}}
\end{aligned}
$$

respects injective objects;

iii. the global section functor $\Gamma$ respects injective objects;

iv. given a coherent sheaf $C=\left(C_{n}\right)_{n}$ the left exact functor

$$
\begin{aligned}
& S h_{\left(A,\left(q_{n}\right)\right)} \longrightarrow \quad \mathbb{N} \text {-projective systems of abelian groups } \\
&\left(S_{n}\right)_{n} \longmapsto \\
&\left.\operatorname{Hom}_{A_{q_{n}}}\left(C_{n}, S_{n}\right)\right)_{n}=\left(\operatorname{Hom}_{A}\left(\Gamma(C), S_{n}\right)_{n} .\right.
\end{aligned}
$$

transforms injective objects into projective systems acyclic for the functor lim; its l-th derived functor is equal to $\left(\operatorname{Ext}_{A_{q_{n}}}^{l}\left(C_{n}, .\right)\right)_{n}$.

Proof: i. The abelian category $S h_{\left(A,\left(q_{n}\right)\right)}$ clearly satisfies $A B 5$ and $A B 3^{*}$. Moreover it is easy to verify that the family of sheaves $\left\{\left(A_{n}^{(m)}\right)_{n}\right\}_{m \in \mathbb{N}}$ with $A_{n}^{(m)}:=0$,

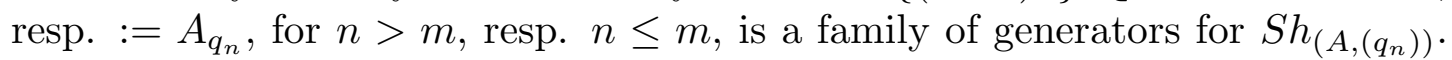
But any abelian category with these properties has enough injective objects (compare [Pop] Thm. 3.10.10).

ii. The functor in question has the exact left adjoint functor $N \longmapsto\left(N_{n}\right)_{n}$ with $N_{n}:=0$ for $n>n_{0}$ and $N:=A_{q_{n}} \otimes_{A_{q_{n_{0}}}} N$ for $n \leq n_{0}$.

iii. Let $J=\left(J_{n}\right)_{n}$ be an injective object in $S h_{\left(A,\left(q_{n}\right)\right)}$. We have to show that, given a left ideal $\mathfrak{l} \subseteq A$, any $A$-module homomorphism $\mathfrak{l} \longrightarrow \Gamma(J)$ extends to an $A$-module homomorphism $A \longrightarrow \Gamma(J)$. It follows from Remark 3.2 that $L:=$ $\left(A_{q_{n}} \otimes_{A} \mathfrak{l}\right)_{n}$ is a coherent subsheaf of $\left(A_{q_{n}}\right)_{n}$. The homomorphism $\mathfrak{l} \longrightarrow \Gamma(J)$ induces homomorphisms $A_{q_{n}} \otimes_{A} \mathfrak{l} \longrightarrow A_{q_{n}} \otimes_{A} \Gamma(J)=J_{n}$ which constitute a

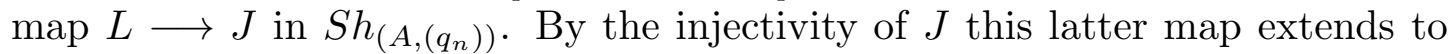
a map $\left(A_{q_{n}}\right)_{n} \longrightarrow J$ which on global sections induces the required extension $A \longrightarrow \Gamma(J)$.

iv. The second part of the assertion is a consequence of ii. To establish the first part we introduce, for any $m \in \mathbb{N}$, the two functors $S \longmapsto S^{(m)}$ and $S \longmapsto S^{[m]}$ from the category $S h_{\left(A,\left(q_{n}\right)\right)}$ into itself defined by

$$
S_{n}^{(m)}:= \begin{cases}S_{m} & \text { if } n \geq m \\ S_{n} & \text { if } n \leq m\end{cases}
$$


and

$$
S_{n}^{[m]}:= \begin{cases}0 & \text { if } n>m \\ S_{n} & \text { if } n \leq m\end{cases}
$$

respectively. The second functor is exact and left adjoint to the first one. Hence the first functor respects injective objects. We consider now a fixed injective object $J=\left(J_{n}\right)_{n}$ in $S h_{\left(A,\left(q_{n}\right)\right)}$. Then the direct product $\prod_{m} J^{(m)}$ in $S h_{\left(A,\left(q_{n}\right)\right)}$ is injective as well. There are obvious sheaf maps $\sigma_{m}: J \longrightarrow J^{(m)}$ and $\sigma_{m+1}^{m}$ : $J^{(m+1)} \longrightarrow J^{(m)}$. We claim that the sequence

$$
0 \rightarrow J \stackrel{\prod \sigma_{m}}{\longrightarrow} \prod_{m} J^{(m)} \stackrel{\prod\left(\mathrm{id}_{J}(m)-\sigma_{m+1}^{m}\right)}{\longrightarrow} \prod_{m} J^{(m)} \longrightarrow 0
$$

is exact. This amounts to the exactness of the sequences

$$
0 \longrightarrow J_{n} \longrightarrow \prod_{m<n} J_{m} \times \prod_{m \geq n} J_{n} \longrightarrow \prod_{m<n} J_{m} \times \prod_{m \geq n} J_{n} \longrightarrow 0
$$

for each $n \in \mathbb{N}$ which is equivalent to the obvious fact that the projective systems

$$
\ldots \stackrel{=}{\longrightarrow} J_{n} \stackrel{=}{\longrightarrow} \ldots \stackrel{=}{\longrightarrow} J_{n} \longrightarrow J_{n-1} \longrightarrow \ldots \longrightarrow J_{1}
$$

have $J_{n}$ as projective limit and have vanishing $\lim ^{(1)}$-term. Applying the functor $\operatorname{Hom}_{S h_{\left(A,\left(q_{n}\right)\right)}}(C,$.$) to the above short exact sequence of injective sheaves we$ obtain the short exact sequence

$$
0 \rightarrow \operatorname{Hom}_{S h_{\left(A,\left(q_{n}\right)\right)}}(C, J) \rightarrow \prod_{m} \operatorname{Hom}_{A_{q_{m}}}\left(C_{m}, J_{m}\right) \rightarrow \prod_{m} \operatorname{Hom}_{A_{q_{m}}}\left(C_{m}, J_{m}\right) \rightarrow 0
$$

It is a well known fact that the surjectivity of the second map is equivalent to the vanishing of $\lim ^{(1)}$ and hence the acyclicity for $\lim _{\longleftarrow}$ of the projective system $\left(\operatorname{Hom}_{A_{q_{m}}}\left(C_{m}, J_{m}\right)\right)_{m}$.

Lemma 8.2: For any $C=\left(C_{n}\right)_{n} \in \operatorname{Coh}_{\left(A,\left(q_{n}\right)\right)}$ and $S=\left(S_{n}\right)_{n} \in S h_{\left(A,\left(q_{n}\right)\right)}$ we have

$$
\begin{aligned}
\operatorname{Hom}_{A}(\Gamma(C), \Gamma(S)) & =\underset{\leftarrow}{\lim } \operatorname{Hom}_{A}\left(\Gamma(C), S_{n}\right) \\
& =\underset{\longleftarrow}{\lim _{n}} \operatorname{Hom}_{A_{q_{n}}}\left(A_{q_{n}} \otimes_{A} \Gamma(C), S_{n}\right) \\
& =\operatorname{Hom}_{S h_{\left(A,\left(q_{n}\right)\right)}}(C, S) .
\end{aligned}
$$

Proof: This is obvious from $C_{n}=A_{q_{n}} \otimes_{A} \Gamma(C)$. 
Lemma 8.3: $i$. For any two coherent sheaves $C=\left(C_{n}\right)_{n}$ and $\widetilde{C}=\left(\widetilde{C}_{n}\right)_{n}$ we have

$$
\operatorname{Ext}_{S h_{\left(A,\left(q_{n}\right)\right)}^{*}}^{*}(C, \widetilde{C}) \stackrel{\rightrightarrows}{\rightarrow} \operatorname{Ext}_{A}^{*}(\Gamma(C), \Gamma(\widetilde{C}))
$$

ii. for any $C=\left(C_{n}\right)_{n} \in \operatorname{Coh}_{\left(A,\left(q_{n}\right)\right)}$ and $S=\left(S_{n}\right)_{n} \in S h_{\left(A,\left(q_{n}\right)\right)}$ we have the short exact sequence

$$
0 \rightarrow \underset{\lim _{n}}{(1)} \operatorname{Ext}_{A_{q_{n}}}^{*-1}\left(C_{n}, S_{n}\right) \rightarrow \operatorname{Ext}_{S h_{\left(A,\left(q_{n}\right)\right)}^{*}}^{*}(C, S) \rightarrow \underset{\underbrace{}_{n}}{\lim } \operatorname{Ext}_{A_{q_{n}}}^{*}\left(C_{n}, S_{n}\right) \rightarrow 0
$$

Proof: Let $\widetilde{C} \longrightarrow J$ be an injective resolution in $S h_{\left(A,\left(q_{n}\right)\right)}$. Since coherent sheaves are acyclic for the global section functor (Thm. B) the sequence

$$
\Gamma(\widetilde{C}) \longrightarrow \Gamma\left(J^{\cdot}\right)
$$

of global sections is exact. As a consequence of Lemma 8.1.iii it then is an injective resolution of the $A$-module $\Gamma(\widetilde{C})$. Using Lemma 8.2 we obtain

$$
\begin{aligned}
\operatorname{Ext}_{S h_{\left(A,\left(q_{n}\right)\right)}}^{*}(C, \widetilde{C}) & =h^{*}\left(\operatorname{Hom}_{S h_{\left(A,\left(q_{n}\right)\right)}}\left(C, J^{*}\right)\right)=h^{*}\left(\operatorname{Hom}_{A}\left(\Gamma(C), \Gamma\left(J^{*}\right)\right)\right) \\
& =\operatorname{Ext}_{A}^{*}(\Gamma(C), \Gamma(\widetilde{C}))
\end{aligned}
$$

This establishes the first assertion. For the second one we note that, by Lemma 8.1.iv, the last identity in Lemma 8.2 gives rise to a spectral sequence

$$
E_{2}^{l, m}=\underset{\lim _{n}^{(l)}}{\left(\operatorname{Ext}_{A_{q_{n}}}^{m}\right.}\left(C_{n}, S_{n}\right) \Rightarrow \operatorname{Ext}_{S h_{\left(A,\left(q_{n}\right)\right)}}^{l+m}(C, S)
$$

Since the higher derived functors of the projective limit vanish for $l \geq 2$ this spectral sequence degenerates into the asserted short exact sequence.

Our dimension formalism will be based on the properties of the functors

$$
\begin{aligned}
\operatorname{Ext}_{A}^{l}(., A): \operatorname{Mod}(A) & \longrightarrow \operatorname{Mod}^{r}(A) \\
M & \longmapsto \operatorname{Ext}_{A}^{l}(M, A)
\end{aligned}
$$

for $l \geq 0$.

Lemma 8.4: For any coadmissible $A$-module $M$ and any integer $l \geq 0$ the $A$-module $\operatorname{Ext}_{A}^{l}(M, A)$ is coadmissible with

$$
\operatorname{Ext}_{A}^{l}(M, A) \otimes_{A} A_{q_{n}}=\operatorname{Ext}_{A_{q_{n}}}^{l}\left(A_{q_{n}} \otimes_{A} M, A_{q_{n}}\right)
$$

for any $n \in \mathbb{N}$. 
Proof: We first claim that

$$
\operatorname{Ext}_{A_{q_{n+1}}}^{l}\left(A_{q_{n+1}} \otimes_{A} M, A_{q_{n+1}}\right) \otimes_{A_{q_{n+1}}} A_{q_{n}}=\operatorname{Ext}_{A_{q_{n}}}^{l}\left(A_{q_{n}} \otimes_{A} M, A_{q_{n}}\right) .
$$

But using a resolution of the $A_{q_{n+1}}$-module $A_{q_{n+1}} \otimes_{A} M$ by finitely generated free $A_{q_{n+1}}$-modules together with the flatness of $A_{q_{n}}$ over $A_{q_{n+1}}$ this reduces to the obvious equality

$$
\operatorname{Hom}_{A_{q_{n+1}}}\left(A_{q_{n+1}}, A_{q_{n+1}}\right) \otimes_{A_{q_{n+1}}} A_{q_{n}}=\operatorname{Hom}_{A_{q_{n}}}\left(A_{q_{n}}, A_{q_{n}}\right) .
$$

It follows that $\left(\operatorname{Ext}_{A_{q_{n}}}^{l}\left(A_{q_{n}} \otimes_{A} M, A_{q_{n}}\right)\right)_{n}$, for each $l \geq 0$, is a coherent sheaf. Moreover, Lemma 8.3 and Theorem B imply that

$$
\operatorname{Ext}_{A}^{l}(M, A) \stackrel{\cong}{\longrightarrow} \Gamma\left(\left(\operatorname{Ext}_{A_{q_{n}}}^{l}\left(A_{q_{n}} \otimes_{A} M, A_{q_{n}}\right)_{n}\right) .\right.
$$

This isomorphism is $A$-linear as can be seen by computing both sides using a projective resolution of the $A$-module $M$ (and observing that $A_{q_{n}}$ is flat over $A$ ). It remains to apply Cor. 3.1.

At this point we briefly recall the notion of Auslander regularity (compare [Bjo] or [LVO] Chap. III). Let $R$ be an arbitrary associative unital ring. The grade of a (left or right) $R$-module $N$ is defined by

$$
j_{R}(N):=\min \left\{l \geq 0: \operatorname{Ext}_{R}^{l}(N, R) \neq 0\right\} .
$$

The $R$-module $N$ is called pure if $\operatorname{Ext}_{R}^{l}\left(\operatorname{Ext}_{R}^{l}(N, R), R\right)=0$ for any $l \neq j_{R}(N)$. Suppose now that $R$ is left and right noetherian. If $N \neq 0$ is finitely generated then its grade $j_{R}(N)$ is bounded above by the projective dimension of $N$. One says that $N$ satisfies the Auslander condition if, for each $l \geq 0$ and any submodule $L \subseteq \operatorname{Ext}_{R}^{l}(N, R)$, we have $j_{R}(L) \geq l$. The noetherian ring $R$ is called (left and right) Auslander regular if every finitely generated left or right $R$-module has finite projective dimension and satisfies the Auslander condition. Suppose henceforth that $R$ is Auslander regular, and let $N$ be a finitely generated $R$ module of projective dimension $d(N)$. For any submodule $N_{0} \subseteq N$ we have

$$
j_{R}(N)=\min \left(j_{R}\left(N_{0}\right), j_{R}\left(N / N_{0}\right)\right) .
$$

Most importantly, $N$ carries a natural filtration, called the dimension filtration, by submodules

$$
N=\Delta^{0}(N) \supseteq \Delta^{1}(N) \supseteq \ldots \supseteq \Delta^{d(N)+1}(N)=0 .
$$

This filtration is characterized by the property that a submodule $L \subseteq N$ has grade $j_{R}(L) \geq l$ if and only if $L \subseteq \Delta^{l}(N)$. In addition one has: 
$-j_{R}(N)=\sup \left\{l \geq 0: \Delta^{l}(N)=N\right\}$

- if $N$ is pure then $N=\Delta^{j_{R}(N)}(N) \supseteq \Delta^{j_{R}(N)+1}(N)=0$;

$-\Delta^{l}(N) / \Delta^{l+1}(N)$ is zero or pure of grade $l$.

These properties show that over an Auslander regular ring the grade can be viewed as a substitute for the codimension of a finitely generated module.

Going back to our Fréchet-Stein algebra $A$ we assume from now on that there is an integer $d \geq 0$ such that each $A_{q_{n}}$ is Auslander regular of a global dimension $\leq d$.

As an immediate consequence of Lemma 8.4 we obtain for any coadmissible $A$-module $M$ that:

$-j_{A_{q_{n+1}}}\left(A_{q_{n+1}} \otimes_{A} M\right) \leq j_{A_{q_{n}}}\left(A_{q_{n}} \otimes_{A} M\right)$ for any $n \in \mathbb{N}$;

- $j_{A}(M)=\min _{n} j_{A_{q_{n}}}\left(A_{q_{n}} \otimes_{A} M\right)$;

$-j_{A}(M) \leq d$ if $M \neq 0$.

In view of Lemma 3.6 it further follows that

$$
j_{A}(M)=\min \left(j_{A}(N), j_{A}(M / N)\right) \text { for any coadmissible submodule } N \subseteq M .
$$

Because of Lemma 8.4 we may apply the latter to any coadmissible submodule $L \subseteq \operatorname{Ext}_{A}^{l}(M, A)$ and obtain

$$
\begin{aligned}
j_{A}(L) & \geq j_{A}\left(\operatorname{Ext}_{A}^{l}(M, A)\right)=\min _{n} j_{A_{q_{n}}}\left(\operatorname{Ext}_{A}^{l}(M, A) \otimes_{A} A_{q_{n}}\right) \\
& =\min _{n} j_{A_{q_{n}}}\left(\operatorname{Ext}_{A_{q_{n}}}^{l}\left(A_{q_{n}} \otimes_{A} M, A_{q_{n}}\right)\right) \\
& \geq l .
\end{aligned}
$$

This means that in an obvious categorical sense any object in the category $\mathcal{C}_{A}$ satisfies the Auslander condition.

Remark 8.5: For any two $M_{1}, M_{2} \in \mathcal{C}_{A}$ we have

$$
\operatorname{Ext}_{A}^{l}\left(M_{1}, M_{2}\right)=0 \quad \text { for } l>d+1 ;
$$

if $M_{2}$ is finitely generated then

$$
\operatorname{Ext}_{A}^{d+1}\left(M_{1}, M_{2}\right)=0 .
$$


Proof: The first vanishing statement is immediate from Lemma 8.3. For the second one we suppose that $M_{2}$ is finitely generated. Writing $M_{2}$ as a quotient of a finitely generated free module and using the long exact Ext-sequence together with additivity reduces us to the case $M_{2}=A$ which is a consequence of Lemma 8.4 .

The possibility of extending the dimension filtration to coadmissible $A$-modules relies on the following fact.

Lemma 8.6: Let $R_{0} \longrightarrow R_{1}$ be a unital homomorphism between two Auslander regular noetherian rings such that $R_{1}$ is flat as a left as well as a right $R_{0}$-module; for any finitely generated (left or right) $R_{0}$-module $N$ we have

$$
\Delta^{l}\left(R_{1} \otimes_{R_{0}} N\right)=R_{1} \otimes_{R_{0}} \Delta^{l}(N) \quad \text { for any } l \geq 0 .
$$

Proof: There is a convergent spectral sequence (see [Bjo] or [LVO]) with $E_{2^{-}}$ term $E_{2}^{l, m}:=\operatorname{Ext}_{R_{0}}^{l}\left(\operatorname{Ext}_{R_{0}}^{-m}\left(N, R_{0}\right), R_{0}\right)$ and abutment $E^{l+m}:=N$ for $l+m=$ 0 , resp. $:=0$ for $l+m \neq 0$. The filtration on $N$ induced by this spectral sequence coincides with the dimension filtration. By using projective resolutions by finitely generated projective modules it follows from our flatness assumption that

$$
R_{1} \otimes_{R_{0}} \operatorname{Ext}_{R_{0}}^{l}\left(\operatorname{Ext}_{R_{0}}^{-m}\left(N, R_{0}\right), R_{0}\right)=\operatorname{Ext}_{R_{1}}^{l}\left(\operatorname{Ext}_{R_{1}}^{-m}\left(R_{1} \otimes_{R_{0}} N, R_{1}\right), R_{1}\right) .
$$

In fact the base extension $R_{1} \otimes_{R_{0}}$. transforms the whole spectral sequence and hence the dimension filtration for the $R_{0}$-module $N$ into the corresponding spectral sequence and dimension filtration for the $R_{1}$-module $R_{1} \otimes_{R_{0}} N$.

Lemma 8.6 implies that any coadmissible $A$-module $M$ carries a dimension filtration

$$
M=\Delta^{0}(M) \supseteq \Delta^{1}(M) \supseteq \ldots \supseteq \Delta^{d+1}(M)=0 .
$$

by coadmissible submodules $\Delta^{l}(M)$ such that

$$
A_{q_{n}} \otimes_{A} \Delta^{l}(M)=\Delta^{l}\left(A_{q_{n}} \otimes_{A} M\right) \quad \text { for any } n \in \mathbb{N} \text {. }
$$

Proposition 8.7: Let $M$ be a coadmissible A-module; we then have:

i. A coadmissible submodule $N \subseteq M$ has grade $j_{A}(N) \geq l$ if and only if $N \subseteq$ $\Delta^{l}(M)$;

ii. $j_{A}(M)=\sup \left\{l \geq 0: \Delta^{l}(M)=M\right\}$; 
iii. if $M$ is pure then $M=\Delta^{j_{A}(M)}(M) \supseteq \Delta^{j_{A}(M)+1}(M)=0$; iv. $\Delta^{l}(M) / \Delta^{l+1}(M)$ is zero or pure of grade $l$.

Proof: i. If $N \subseteq \Delta^{l}(M)$ then $j_{A}(N) \geq j_{A}\left(\Delta^{l}(M)\right)=\min j_{A_{q_{n}}}\left(\Delta^{l}\left(A_{q_{n}} \otimes_{A} M\right)\right) \geq$ l. On the other hand, if $j_{A}(N) \geq l$ then $j_{A_{q_{n}}}\left(A_{q_{n}} \otimes_{A} N\right) \geq l$ for any $n$, hence $A_{q_{n}} \otimes_{A} N \subseteq \Delta^{l}\left(A_{q_{n}} \otimes_{A} M\right)$ for any $n$, and therefore $N \subseteq \Delta^{l}(M)$. ii. This is a formal consequence of the first assertion. iii. We have $j_{A}(M)=$ $j_{A_{q_{n}}}\left(A_{q_{n}} \otimes_{A} M\right)$ for $n \in \mathbb{N}$ big enough. Lemma 8.4 then implies, for these $n$, that $A_{q_{n}} \otimes_{A} M$ is pure and hence that $A_{q_{n}} \otimes_{A} M=\Delta^{j_{A}(M)}\left(A_{q_{n}} \otimes_{A} M\right) \supseteq$ $\Delta^{j_{A}(M)+1}\left(A_{q_{n}} \otimes_{A} M\right)=0$. iv. Suppose that $\Delta^{l}(M) / \Delta^{l+1}(M)$ is nonzero. According to Lemma 8.4 it suffices to show that $A_{q_{n}} \otimes_{A}\left(\Delta^{l}(M) / \Delta^{l+1}(M)\right)$ is pure of grade $l$ for any $n \in \mathbb{N}$ big enough. Since $A_{q_{n}}$ is flat over $A$ (Remark 3.2) we have $A_{q_{n}} \otimes_{A}\left(\Delta^{l}(M) / \Delta^{l+1}(M)\right)=\left(A_{q_{n}} \otimes_{A} \Delta^{l}(M)\right) /\left(A_{q_{n}} \otimes_{A} \Delta^{l+1}(M)\right)=$ $\Delta^{l}\left(A_{q_{n}} \otimes_{A} M\right) / \Delta^{l+1}\left(A_{q_{n}} \otimes_{A} M\right)$. By assumption the left hand side is nonzero for big $n$. If so the right hand side is pure of grade $l$.

All these properties confirm our claim that under the assumption (DIM) the grade can be used as a well behaved codimension function on the category $\mathcal{C}_{A}$.

Before we show that all this applies to the algebra $D(G, K)$ for a compact locally $\mathbb{Q}_{p}$-analytic group $G$ we recall, for the convenience of the reader, the following facts from general ring theory.

Lemma 8.8: Let $R_{0} \longrightarrow R_{1}$ be a unital homomorphism of (left or right) noetherian rings; suppose that there are units $b_{1}=1, b_{2}, \ldots, b_{m} \in\left(R_{1}\right)^{\times}$which form a basis of $R_{1}$ as an $R_{0}$-module and which satisfy:

- $b_{i} R_{0}=R_{0} b_{i}$ for any $1 \leq i \leq m$,

- for any $1 \leq i, j \leq m$ there is a $1 \leq k \leq m$ such that $b_{i} b_{j} \in b_{k} R_{0}$, and

- for any $1 \leq i \leq m$ there is a $1 \leq l \leq m$ such that $b_{i}^{-1} \in b_{l} R_{0}$.

We then have

$(+) \operatorname{Ext}_{R_{1}}^{*}\left(N_{1}, R_{1} \otimes_{R_{0}} N_{0}\right)$, resp. $\operatorname{Ext}_{R_{1}}^{*}\left(N_{1}, N_{0} \otimes_{R_{0}} R_{1}\right) \cong \operatorname{Ext}_{R_{0}}^{*}\left(N_{1}, N_{0}\right)$

for any pair of finitely generated left, resp. right, $R_{i}$-modules $N_{i}(i=0,1)$. If $N$ is any finitely generated (left or right) $R_{1}$-module then:

i. $\operatorname{Ext}_{R_{1}}^{*}\left(N, R_{1}\right) \cong \operatorname{Ext}_{R_{0}}^{*}\left(N, R_{0}\right)$ and in particular $j_{R_{1}}(N)=j_{R_{0}}(N)$;

ii. the projective dimensions of $N$ as an $R_{1}$-module and as an $R_{0}$-module coincide; in particular, $N$ is projective as an $R_{1}$-module if and only if it is projective as an $R_{0}$-module.

Finally, the rings $R_{0}$ and $R_{1}$ have the same global dimension.

Proof: The case of right modules being analogous we only consider the case of left modules in the following. Let $\ell: R_{1} \longrightarrow R_{0}$ denote the projection onto the first summand in the decomposition

$$
R_{1}=\bigoplus_{i} b_{i} R_{0}=\bigoplus_{i} R_{0} b_{i}
$$


It induces a map between the two sides in $(+)$. By using a projective resolution of the $R_{1}$-module $N_{1}$ by finitely generated free $R_{1}$-modules (which as a consequence of our assumptions also is such a resolution for $N_{1}$ as an $R_{0}$-module) it suffices to consider the case $*=0$ and $N_{1}=R_{1}$. By using a presentation of $N_{0}$ by finitely generated free $R_{0}$-modules we are further reduced to show that the map

$$
\begin{aligned}
\operatorname{Hom}_{R_{1}}\left(R_{1}, R_{1}\right) & \longrightarrow \operatorname{Hom}_{R_{0}}\left(R_{1}, R_{0}\right) \\
\Phi & \longmapsto \ell \circ \Phi
\end{aligned}
$$

is bijective. We have

$$
\Phi(a)=b_{1}(\ell \circ \Phi)\left(b_{1}^{-1} a\right)+\ldots+b_{m}(\ell \circ \Phi)\left(b_{m}^{-1} a\right) \quad \text { for any } a \in R_{1}
$$

which proves the injectivity of the map. To establish surjectivity we suppose given a left $R_{0}$-module map $\Psi: R_{1} \longrightarrow R_{0}$. It is straightforward to check that

$$
\Phi(a):=b_{1} \Psi\left(b_{1}^{-1} a\right)+\ldots+b_{m} \Psi\left(b_{m}^{-1} a\right)
$$

in fact is a preimage of $\Psi$. This proves the isomorphism $(+)$.

The statement i. is $(+)$ for $N_{0}=R_{0}$. In the statement ii. we first consider the particular case. If $N$ is projective as an $R_{1}$-module then obviously also as an $R_{0}$-module since $R_{1}$ is finitely generated free over $R_{0}$. Suppose therefore that $N$ is projective over $R_{0}$. We have to show that $\operatorname{Ext}_{R_{1}}^{1}(N, X)=0$ for all $R_{1}$-modules $X$. Since $R_{1}$ is noetherian and $N$ is finitely generated the functor $\operatorname{Ext}_{R_{1}}^{1}(N,$. commutes with filtered inductive limits. Hence it suffices to consider finitely generated modules $X$. From $(+)$ we then have that $\operatorname{Ext}_{R_{1}}^{1}\left(N, R_{1} \otimes_{R_{0}} X\right)=0$. But $X$ as an $R_{1}$-module is a direct summand of $R_{1} \otimes_{R_{0}} X$ since $R_{0}$ is a direct $R_{0^{-}}$ module summand of $R_{1}$. The general assertion about the equality of projective dimensions as well as the asserted equality of global dimensions is easily deduced from this using appropriate projective resolutions (compare [MCR] Thm. 7.5.6).

Theorem 8.9: For any compact locally $\mathbb{Q}_{p}$-analytic group $G$ the Fréchet-Stein algebra $D(G, K)$ satisfies $(D I M)$ with $d:=\operatorname{dim}(G)$.

Proof: In a first step we assume in addition that $G$ is a uniform pro- $p$-group. The structure of $D(G, K)$ as a two sided Fréchet-Stein algebra is given, according to section 4 , by the Banach algebras $D_{r}(G, K)$ where $1 / p<r<1, r \in p^{\mathbb{Q}}$. We have to show that each $D_{r}(G, K)$ is Auslander regular of global dimension $\leq d$. The filtration on $D_{r}(G, K)$ derived from the submultiplicative norm \|\|$_{r}$ is quasi-integral. From Thm. 4.5.i we know that the associated graded ring $g r_{r} D_{r}(G, K)$ is isomorphic to a polynomial ring $k\left[X_{0}, X_{0}^{-1}, X_{1}, \ldots, X_{d}\right]$ over the residue class field $k$ of $K$. This is a regular commutative noetherian ring of global dimension $d+1$. By [LVO] Cor. I.7.2.2, Thm. III.2.2.5, and III.2.4.3 it follows that $D_{r}(G, K)$ is Auslander regular of global dimension $\leq d+1$. To obtain the stronger bound $\leq d$ we have to reexamine part of this reasoning more 
closely. By [MCR] 7.2.6 we may replace $K$ by a finite extension. This allows us, by the computation in the proof of Lemma 4.8, to assume that $g r_{r}^{\cdot} F_{r}^{0} D_{r}(G, K)$ is isomorphic to a polynomial ring $k\left[Y_{0}, \ldots, Y_{d}\right]$. Hence the global dimension of $F_{r}^{0} D_{r}(G, K)$ also is $\leq d+1$. On the other hand since $F_{r}^{0+} D_{r}(G, K)$ is contained in the Jacobson radical of $F_{r}^{0} D_{r}(G, K)$ (see [LVO] Lemma I.3.5.5(2)) multiplication by $p$ is zero on any simple module over $F_{r}^{0} D_{r}(G, K)$. It therefore follows from $[\mathrm{MCR}]$ 7.4.3/4 that the global dimension of $D_{r}(G, K)=\mathbb{Q}_{p} \otimes F_{r}^{0} D_{r}(G, K)$ is $\leq d$.

For general $G$ we choose an open normal subgroup $H \subseteq G$ which is a uniform pro-p-group. In view of the construction of the algebras $D_{r}(G, K)$ in the proof of Thm. 5.1 the ring extensions $D_{r}(H, K) \subseteq D_{r}(G, K)$ satisfy the assumptions of Lemma 8.8. It follows that with $D_{r}(H, K)$ also $D_{r}(G, K)$ is Auslander regular of global dimension $\leq d$.

Remark 8.10: Let $H$ be an open subgroup in the compact locally $\mathbb{Q}_{p}$-analytic group $G$; for any coadmissible $D(G, K)$-module $M$ we have

$$
j_{D(G, K)}(M)=j_{D(H, K)}(M)
$$

Proof: This follows from Lemma 8.8 applied to the ring extensions $D_{r}\left(H_{1}, K\right) \subseteq$ $D_{r}(H, K)$ and $D_{r}\left(H_{1}, K\right) \subseteq D_{r}(G, K)$ provided we find a uniform pro- $p$-group $H_{1}$ contained in $H$ which is open normal in $G$. But it is a straightforward consequence of [DDMS] Thm. 4.2 and the discussion before Thm. 4.9 that $G$ has a fundamental system of open characteristic subgroups which are uniform pro- $p$.

We finally consider an arbitrary locally $\mathbb{Q}_{p}$-analytic group $G$ of dimension $d$ and a coadmissible (left) $D(G, K)$-module $M$. Remark 8.10 implies that, for any two compact open subgroups $H, H^{\prime} \subseteq G$, we have

$$
j_{D(H, K)}(M)=j_{D\left(H^{\prime}, K\right)}(M) .
$$

This fact allows us to unambiguously define the codimension of $M$ by

$$
\operatorname{codim}(M):=j_{D(H, K)}(M)
$$

Similarly, by Remark 8.10 and Prop. 8.7, the dimension filtration

$$
M=\Delta^{0}(M) \supseteq \Delta^{1}(M) \supseteq \ldots \supseteq \Delta^{d+1}(M)=0 .
$$

of $M$ as a $D(H, K)$-module does not depend on the choice of $H$. 
Proposition 8.11: For any coadmissible $D(G, K)$-module $M$ we have:

i. Each $\Delta^{l}(M)$ is a coadmissible $D(G, K)$-submodule of $M$;

ii. a coadmissible $D(G, K)$-submodule $N \subseteq M$ has codimension $\geq l$ if and only if $N \subseteq \Delta^{l}(M)$;

iii. $\operatorname{codim}(M)=\sup \left\{l \geq 0: \Delta^{l}(M)=M\right\}$;

$i v$. all nonzero coadmissible $D(G, K)$-submodules of $\Delta^{l}(M) / \Delta^{l+1}(M)$ have codimension $l$.

Proof: Everything except the $D(G, K)$-invariance of $\Delta^{l}(M)$ is a consequence of Prop. 8.7. Since $D(G, K)$ as a $D(H, K)$-module is generated by the $\delta_{g}$ for $g \in G$ it suffices to show that $g\left(\Delta^{l}(M)\right) \subseteq \Delta^{l}(M)$. The map $\Delta^{l}(M) \stackrel{g}{\longrightarrow} g\left(\Delta^{l}(M)\right)$ is a module isomorphism relative to the algebra isomorphism $\delta_{g} \cdot \delta_{g^{-1}}: D(H, K) \stackrel{\cong}{\longrightarrow}$ $D\left(g H g^{-1}, K\right)$. It follows that $l \leq j_{D(H, K)}\left(\Delta^{l}(M)\right)=j_{D\left(g H g^{-1}, K\right)}\left(g\left(\Delta^{l}(M)\right)\right.$. Since the dimension filtration is independent of the choice of the compact open subgroup we obtain $g\left(\Delta^{l}(M)\right) \subseteq \Delta^{l}(M)$.

Definition: A coadmissible $D(G, K)$-module $M$ with $\operatorname{codim}(M) \geq \operatorname{dim}(G)$ is called zero-dimensional.

A nonzero zero-dimensional $D(G, K)$-module $M$ satisfies $\operatorname{codim}(M)=\operatorname{dim}(G)$.

Theorem 8.12: Let $G$ be a locally $\mathbb{Q}_{p}$-analytic group and let $M$ be a coadmissible $D(G, K)$-module; if the action of the universal enveloping algebra $U(\mathfrak{g})$ on $M$ is locally finite, i.e., if $U(\mathfrak{g}) x$, for any $x \in M$, is a finite dimensional $\mathbb{Q}_{p}$-vector space then $M$ is zero-dimensional.

Corollary 8.13: If $V$ is an admissible smooth $G$-representation then $V_{b}^{\prime}$ is a zero-dimensional $D(G, K)$-module.

The statement of Thm. 8.12 only depends on an arbitrarily chosen compact open subgroup of $G$. We therefore let $H$ in the following be a compact locally $\mathbb{Q}_{p}$-analytic group which is a uniform pro-p-group. In this case Thm. 8.12 will be a consequence of the following more general, but technical criterion. Recalling some notation from section 4 we fix an ordered basis $\left(h_{1}, \ldots, h_{d}\right)$ of $G$ and let $\psi: \mathbb{Z}_{p}^{d \stackrel{\sim}{\longrightarrow}} G$ given by $\psi\left(\left(x_{1}, \ldots x_{d}\right)\right)=h_{1}^{x_{1}} \cdot \ldots \cdot h_{d}^{x_{d}}$ denote the corresponding global chart. Any distribution $\lambda \in D(H, K)$ has a unique convergent expansion in the elements $b_{1}=h_{1}-1, \ldots, b_{d}=h_{d}-1$. As always we let $r$ vary over the set $\left\{r \in p^{\mathbb{Q}}: 1 / p<r<1\right\}$.

Proposition 8.14: Suppose that $\lambda_{1}, \ldots, \lambda_{d} \in D(H, K)$ are nonzero elements such that, for each $1 \leq i \leq d$, the expansion of $\lambda_{i}$ only involves powers of $b_{i}$, and 
let $J \subseteq D(H, K)$ be the left ideal generated by $\lambda_{1}, \ldots, \lambda_{d}$; then the coadmissible $D(H, K)$-module $D(H, K) / J$ is zero-dimensional.

Proof: Since $J$ is finitely generated the module $D(H, K) / J$ is coadmissible and the corresponding coherent sheaf is given by $D_{r}(H, K) / D_{r}(H, K) J$. To simplify notation we use in the following the abbreviation $D_{r}:=D_{r}(H, K)$. So we have to show that $j_{D_{r}}\left(D_{r} / D_{r} J\right) \geq d$. We equip $D_{r} J$ and $D_{r} / D_{r} J$ with the filtrations induced by the filtration $F_{r}^{\cdot} D_{r}$. Hence $g r_{r}^{\cdot}\left(D_{r} / D_{r} J\right)=\left(g r_{r}^{\cdot} D_{r}\right) /\left(g r_{r} D_{r} J\right)$. Recall from Thm. 4.5.i that $g r_{r} D_{r}=g r^{\prime} K\left[\sigma_{r}\left(b_{1}\right), \ldots, \sigma_{r}\left(b_{d}\right)\right]$ is a polynomial ring in $d$ variables over $g r \cdot K$. According to [LVO] Thm. III.2.5.2 we have $j_{D_{r}}\left(D_{r} / D_{r} J\right)=j_{g r_{r}^{*} D_{r}}\left(g r_{r}^{*}\left(D_{r} / D_{r} J\right)\right)$. We therefore are reduced to showing that $j_{g r_{r} D_{r}}\left(g r_{r} D_{r} / g r_{r} D_{r} J\right) \geq d$. The ring $R:=g r_{r} D_{r}$ is a commutative (Auslander) regular and catenary noetherian domain of Krull dimension $d+1$. For any finitely generated module $N$ over such a ring $R$ standard commutative algebra (compare $[\mathrm{BH}]$ Cor. 3.5.11) implies the formula

$$
j_{R}(N)=d+1-\operatorname{Krulldim}(R / \operatorname{ann}(N))
$$

where $\operatorname{ann}(N)$ denotes the annihilator ideal of $N$ in $R$. Hence it remains to see that the factor ring $g r_{r}^{\cdot} D_{r} / g r_{r}^{\cdot} D_{r} J$ has Krull dimension $\leq 1$. By our assumption on the $\lambda_{i}$ we have a surjection

$$
g r^{\cdot} K\left[\sigma_{r}\left(b_{1}\right)\right] /\left\langle\sigma_{r}\left(\lambda_{1}\right)\right\rangle \underset{g r^{\prime} K}{\otimes} \ldots \underset{g r^{\prime} K}{\otimes} g r^{\cdot} K\left[\sigma_{r}\left(b_{d}\right)\right] /\left\langle\sigma_{r}\left(\lambda_{d}\right)\right\rangle \longrightarrow g r_{r}^{\cdot} D_{r} / g r_{r}^{\cdot} D_{r} J .
$$

Each $\operatorname{gr} K\left[\sigma_{r}\left(b_{i}\right)\right] /\left\langle\sigma_{r}\left(\lambda_{i}\right)\right\rangle$ is finitely generated as a $g r^{\cdot} K$-module. It follows that $g r_{r}^{\cdot} D_{r} / g r_{r}^{\cdot} D_{r} J$ is finite over the one dimensional ring $g r^{\cdot} K$ and therefore has Krull dimension $\leq 1$ (compare $[\mathrm{B}-\mathrm{CA}] \mathrm{V} \S 2.1)$.

Proof of Thm.8.12 : According to Prop. 8.11 we have to show that $M \subseteq$ $\Delta^{d}(M)$. In fact, it suffices to show that, for any $x \in M$, the submodule $N:=D(H, K) x$ of $M$ generated by $x$ (which is coadmissible by Cor. 3.4.iv) is zero-dimensional. We write $N=D(H, K) / J_{1}$ for some left ideal $J_{1} \subseteq D(H, K)$. By assumption $J_{1}$ contains an ideal of finite codimension $J_{0}$ of $U(\mathfrak{g})$. The Lie algebra $\mathfrak{g}$ has the basis $\partial_{1}, \ldots, \partial_{d}$ where $\partial_{i}:=\psi_{*}\left(\left(\partial / \partial x_{i}\right)_{\mid x_{i}=0}\right)=\log \left(1+b_{i}\right)$. Since $J_{0}$ is of finite codimension in $U(\mathfrak{g})$ it contains, for any $1 \leq i \leq d$, a nonzero polynomial $P_{i}\left(\partial_{i}\right) \in K\left[\partial_{i}\right]$. The left ideal $J \subseteq J_{1} \subseteq D(H, K)$ generated by $P_{1}\left(\partial_{1}\right), \ldots, P_{d}\left(\partial_{d}\right)$ satisfies the assumptions of Prop. 8.14. Hence $D(H, K) / J$ and a fortiori its quotient $N$ are zero-dimensional.

The subsequent criterion is obtained by a very similar argument.

Theorem 8.15: Let $G$ be a compact locally $\mathbb{Q}_{p}$-analytic group and let $M$ be a coadmissible $D(G, K)$-module; if the coherent sheaf corresponding to $M$ is a sheaf of finite dimensional $K$-vector spaces then $M$ is zero-dimensional. 
Proof: Using Remark 8.10 and the notations introduced before Prop. 8.14 the assertion reduces to the claim that $j_{D_{r}(H, K)}(M) \geq d$ for any $D_{r}(H, K)$-module $M$ which is finite dimensional as a $K$-vector space. We equip $M$ with a filtration $F^{\cdot} M$ which is good with respect to $F_{r} \cdot D_{r}(H, K)$ and obtain from [LVO] Thm. III.2.5.2 that $j_{D_{r}(H, K)}(M)=j_{g r_{r} D_{r}(H, K)}\left(g r^{\cdot} M\right)$. The assumption that $M$ is finite dimensional over $K$ easily implies that $g r^{\cdot} M$ is finitely generated over $g r \cdot K$. From this point on the rest of the argument is exactly the same as in the proof of Prop. 8.14.

We strongly believe that the results of this section extend to locally $L$-analytic groups $G$ over any finite extension $L$ of $\mathbb{Q}_{p}$. But to establish the Auslander regularity (or possibly a weaker but sufficient Auslander-Gorenstein property compare $[\mathrm{Bjo}]$ ) of the corresponding Banach algebras in this situation seems to require new ideas.

\section{References}

[Ban] Bănică C.: Une caracterisation de la dimension d'un faisceau analitique coherent. Compositio math. 25, 101-108 (1972)

[Bjo] Björk J.-E.: Filtered Noetherian Rings. In Noetherian rings and their applications, Math. Survey Monographs 24, pp. 59-97, AMS 1987

[BGR] Bosch S., Güntzer U., Remmert R: Non-Archimedean Analysis. Berlin-Heidelberg-New York: Springer 1984

[B-CA] Bourbaki N.: Commutative Algebra. Paris: Hermann 1972

[B-GT] Bourbaki N.: General Topology, Chap. 1-4. Berlin-Heidelberg-New York: Springer 1989

[B-TVS] Bourbaki N.: Topological Vector Spaces. Berlin-Heidelberg-New York: Springer 1987

[BH] Bruns W., Herzog J.: Cohen-Macaulay rings. Cambridge Univ. Press 1993

[DDMS] Dixon J.D., du Sautoy M.P.F., Mann A., Segal D.: Analytic Pro-pGroups. Cambridge Univ. Press 1999

[Fea] Féaux de Lacroix C. T.: Einige Resultate über die topologischen Darstellungen $p$-adischer Liegruppen auf unendlich dimensionalen Vektorräumen über einem $p$-adischen Körper. Thesis, Köln 1997, Schriftenreihe Math. Inst. Univ. Münster, 3. Serie, Heft 23, pp. 1-111 (1999)

[For] Forster O.: Zur Theorie der Steinschen Algebren und Moduln. Math. Z. 97, 376-405 (1967)

[EGA] Grothendieck A., Dieudonné J.: Éléments de géométrie algébrique, Chap. III. Publ. Math. IHES 11 (1961) 
[Laz] Lazard M.: Groupes analytiques p-adique. Publ. Math. IHES 26, 389-603 (1965)

[LVO] Li Huishi, van Oystaeyen F.: Zariskian Filtrations. Dordrecht: Kluwer 1996

[MCR] McConnell J.C., Robson J.C.: Noncommutative noetherian rings. Chichester: Wiley 1987

[Pop] Popescu N.: Abelian Categories with Applications to Rings and Modules. London-New York: Academic Press 1973

[NFA] Schneider P.: Nonarchimedean Functional Analysis. Berlin-HeidelbergNew York: Springer 2001

[ST1] Schneider P., Teitelbaum J.: $U(\mathfrak{g})$-finite locally analytic representations. Representation Theory 5, 111-128 (2001)

[ST2] Schneider P., Teitelbaum J.: Locally analytic distributions and $p$-adic representation theory, with applications to $G L_{2}$. J. AMS 15, 443-468 (2002)

[ST3] Schneider P., Teitelbaum J.: Banach space representations and Iwasawa theory. Israel J. Math. 127, 359-380 (2002)

[ST4] Schneider P., Teitelbaum J.: p-adic Fourier theory. Documenta Math. 6, 447-481 (2001)

[ST5] Schneider P., Teitelbaum J.: p-adic boundary values. In Cohomologies $p$-adiques et applications arithmétiques (I) (Eds. Berthelot/Fontaine/ Illusie/Kato/Rapoport), Astérisque 278, 51-125 (2002)

Peter Schneider

Mathematisches Institut

Westfälische Wilhelms-Universität Münster

Einsteinstr. 62

D-48149 Münster, Germany

pschnei@math.uni-muenster.de

http://www.uni-muenster.de/math/u/schneider

Jeremy Teitelbaum

Department of Mathematics, Statistics, and Computer Science (M/C 249)

University of Illinois at Chicago

851 S. Morgan St.

Chicago, IL 60607, USA

jeremy@uic.edu

http://raphael.math.uic.edu/ jeremy 